\title{
A Framework for Robust Stability of Systems Over Finite Alphabets
}

\author{
Danielle C. Tarraf, Member, IEEE, Alexandre Megretski, Senior Member, IEEE, and \\ Munther A. Dahleh, Fellow, IEEE
}

\begin{abstract}
Systems over finite alphabets are discrete-time systems whose input and output signals take their values in finite sets. Three notions of input/output stability (gain stability, incremental stability and external stability) that are particularly applicable to this class of systems are proposed and motivated through examples. New formulations for generalized small gain and incremental small gain theorems are presented, thus showing that gain stability and incremental stability are useful robustness measures. The paper then focuses on deterministic finite state machine (DFM) models. For this class, the problems of verifying gain stability, incremental stability, and corresponding gain bounds are shown to reduce to searching for an appropriate storage function. These problems are also shown to be related to the problem of verifying the nonexistence of negative cost cycles in an appropriately constructed network. Using this insight and based on a solution approach for discrete shortest path problems, a strongly polynomial algorithm is proposed. Finally, incremental stability and external stability are shown to be equivalent notions for this class of systems.
\end{abstract}

Index Terms-Dissipative system, finite state machine, incremental stability, input/output stability, shortest path problem, small gain theorem, storage functions, system over finite alphabet.

\section{INTRODUCTION}

\section{A. Motivation and Scope}

$\mathbf{T}$ HIS paper is motivated by the desire to develop a new paradigm that enables systematic analysis, design and optimization of hybrid systems. Hybrid systems are systems that involve interacting continuous and discrete dynamics ${ }^{1}$ : They have received much attention in the past decade [1]-[8] due to their pervasive presence in engineered [9]-[13] as well as biological systems [14], [15] and the multitude of new challenges that they present. The paper focuses on a class of hybrid systems, which we refer to as "systems over finite alphabets", where the input and output interfaces are discrete. Systems over finite alphabets are discrete-time systems whose input and output signals take

Manuscript received February 15, 2006; revised March 18, 2007 and October 18, 2007. Published August 27, 2008 (projected). Recommended by Associated Editor C. Abdallah. This research was supported by the Air Force Aerospace Research-OSR Grant FA9550-04-1-0052 while the first author was at MIT.

D. C. Tarraf is with the Division of Control and Dynamical Systems at the California Institute of Technology, Pasadena, CA 91125 USA (e-mail: dtarraf@cds. caltech.edu).

A. Megretski and M. A. Dahleh are with the Department of Electrical Engineering and Computer Science at the Massachusetts Institute of Technology, Cambridge, MA 02139 USA (e-mail: ameg@mit.edu; dahleh@mit.edu).

Digital Object Identifier 10.1109/TAC.2008.923658

${ }^{1}$ Continuous and discrete dynamics are understood to mean dynamics that evolve in continuous and discrete state-spaces, respectively. their values in finite sets: This is, for instance, the case where the actuation takes the form of an on/off or a multilevel switch, or when sensing is coarse and quantized.

The paradigm proposed for systems over finite alphabets bases the analysis and control synthesis on an approximate model belonging to a simpler class of systems, while accounting for the approximation error. The classical robust control framework [16], [17] and generalizations of it provide paradigms and efficient computational tools for problems of analysis and optimal controller synthesis for systems that are, in some sense, close to being linear. The idea there is to approximate a given system by a nominal LTI model, and to establish a quantitative measure for the degree of fidelity of the nominal model to the original system. This measure is typically an induced gain bound [17] or an integral quadratic constraint [18] for the error system. An LTI controller is then designed to stabilize the nominal model and to meet other performance objectives, also typically described in terms of induced gain bounds or integral quadratic constraints. Robust performance of the actual closed-loop system is verified using a small gain argument [19] or an S-procedure [20], [21]. An extension of small gain arguments for input-to-sate stability analysis of hybrid systems was recently proposed in [22], in which the hybrid system was decomposed into the feedback interconnection of its discrete and continuous subsystems for the purpose of analysis.

While the traditional robust control approaches have been successfully used in a variety of settings, they become inadequate when the systems in question are highly nonlinear or hybrid (particularly when the inputs and outputs are restricted to finite sets), partially due to their reliance on LTI nominal models. For such problems, we seek an alternative robust control framework in which the hybrid systems are approximated by nominal finite state machine models for the purpose of analysis and controller synthesis. This finite state machine based approach, which was first proposed in [23], seems natural for two reasons: 1) the computer-based implementation of controllers is discrete and 2) dynamical systems can be thought of as infinite state machines, with finite state machines as their obvious approximation.

Developing a finite state machine based robust control framework necessitates results in three complementary directions: 1) approaches for generating approximate finite state machine models of hybrid systems, with useful guarantees on the quality of approximation, 2) a set of constructive tools for robust performance analysis, and 3) tools for synthesizing finite state machine controllers. This paper focuses exclusively on item 2) in the above list. Interested readers are referred to [24]-[32] for 
an overview of recent advances in addressing item 1), to [23], [24], [33] for several related treatments of item 3), and to [34] for an exposition of a new approach for controller synthesis for a class of switched systems that utilizes the results of this paper and the aforementioned references.

The first part of this paper (Section II) develops a formal framework for robust stability analysis that is, in particular, applicable to systems over finite alphabets. The components of this framework are several notions of input/output stability and corresponding generalized small gain theorems. Standard system descriptions in terms of induced norms or integral quadratic constraints cannot generally be used in this setting since the alphabet sets are arbitrary finite sets with no $a$ priori assumptions on their algebraic or topological structure. The second part of this paper focuses exclusively on a class of systems over finite alphabets described by finite state machine models. Analytical (Section III) and computational (Section IV) tools for stability analysis of this class of systems are presented.

\section{B. Notation}

The following notation is used throughout the paper: $\operatorname{card}(\mathcal{A})$ denotes the cardinality of set $\mathcal{A}$. Given two sets $\mathcal{A}$ and $\mathcal{B}, \mathcal{A} \times \mathcal{B}$ denotes their Cartesian product. $\mathbb{Z}_{+}$and $\mathbb{R}_{+}$denote the sets of non-negative integers and non-negative reals, respectively. For every $k \in \mathbb{Z}_{+}, \mathbb{Z}_{k}=\left\{i \in \mathbb{Z}_{+} \mid i \leq k\right\}$. $\mathcal{A}^{\mathbb{Z}_{+}}$is the set of all infinite sequences over $\mathcal{A}$; that is, $\mathcal{A}^{\overline{\mathbb{Z}}_{+}}=\left\{h: \mathbb{Z}_{+} \rightarrow \mathcal{A}\right\}$. Likewise, $\mathcal{A}^{\mathbb{Z}_{k}}$ is the set of all length $k+1$ sequences over $\mathcal{A} ; \mathcal{A}^{\mathbb{Z}_{k}}=$ $\left\{h: \mathbb{Z}_{k} \rightarrow \mathcal{A}\right\}$. An element of $\mathcal{A}$ is denoted by $a$ while an element of $\mathcal{A}^{\mathbb{Z}_{+}}\left(\mathcal{A}^{\mathbb{Z}_{k}}\right)$ is denoted by a or $\{a(t)\}_{t=0}^{\infty}\left(\{a(t)\}_{t=0}^{k}\right)$. For $a \in \mathcal{A}^{p}, a_{i}$ denotes the $i$ th component of $a$. The notation $\subseteq$ and $\subset$ denotes inclusion and proper inclusion, respectively. For $\mathcal{A}_{o} \subset \mathcal{A}, \mathbf{I}_{\mathcal{A}_{o}}$ denotes the indicator function of set $\mathcal{A}_{o}$, that is the function $\mathbf{I}_{\mathcal{A}_{o}}: \mathcal{A} \rightarrow\{0,1\}$ defined by $\mathbf{I}_{\mathcal{A}_{o}}(a)=1$ for $a \in \mathcal{A}_{o}$ and $\mathbf{I}_{\mathcal{A}_{o}}(a)=0$, otherwise.

\section{SySTEMS AND STABILITY: A FoRMAL FRAMEWORK}

\section{A. Systems Over Finite Alphabets}

A discrete-time signal is understood to be an infinite sequence over some prescribed set, which we refer to as alphabet set.

Definition 1: A discrete-time system $S$ is a set of pairs of signals, $S \subset \mathcal{U}^{\mathbb{Z}_{+}} \times \mathcal{Y}^{\mathbb{Z}_{+}}$.

A system is thus a process characterized by its feasible signals set, which is simply a list of ordered pairs of all the signals (sequences over input alphabet set $\mathcal{U}$ ) that can be applied as an input to this process, and all the output signals (sequences over output alphabet set $\mathcal{Y}$ ) that can be potentially exhibited by the process in response to each of the input signals. Throughout the paper, the same symbol will be used interchangeably to denote a system and its feasible signals set.

Example 1: The system $S$ defined by the feasible signals set

$$
S=\left\{(\mathbf{u}, \mathbf{y}) \in \mathbb{R}^{\mathbb{Z}_{+}} \times \mathbb{R}^{\mathbb{Z}_{+}} \mid y(t)=k u(t), \forall t \in \mathbb{Z}_{+}\right\}
$$

is a memoryless system, gain $k$.

The case where signals are defined over finite alphabet sets is of particular interest in this paper.

Definition 2: A system over finite alphabets is a discrete-time system whose input and output alphabet sets are finite.
Example 2: The system over finite alphabets defined by the feasible signals set

$$
S=\left\{(\mathbf{u}, \mathbf{y}) \in \mathcal{U}^{\mathbb{Z}_{+}} \times \mathcal{Y}^{\mathbb{Z}_{+}} \mid y(t)=k u(t), \forall t \in \mathbb{Z}_{+}\right\}
$$

where $\mathcal{U}=\{-1,0,1\}$ and $\mathcal{Y}=\{-k, 0, k\}$ is a memoryless system, gain $k$, whose input is restricted to three values: $-1,0$, and 1 , and whose output is hence also restricted to three values: $-k, 0$, and $k$.

\section{B. Notions of Input-Output Stability}

Three notions of input/output stability are proposed in this section: Gain stability, incremental stability and external stability. Note that while these notions are defined for arbitrary systems, they are particularly of interest for systems over finite alphabets. A discussion of their significance and their relations to existing notions of stability is deferred until Section II-D, with a set of illustrative examples following in Section II-E.

Definition 3: Consider a system $S \subset \mathcal{U}^{\mathbb{Z}_{+}} \times \mathcal{Y}^{\mathbb{Z}_{+}}$and let $\rho: \mathcal{U} \rightarrow \mathbb{R}$ and $\mu: \mathcal{Y} \rightarrow \mathbb{R}$ be given functions. $S$ is $\rho / \mu$ gain stable if there exists a finite non-negative constant $\gamma$ such that the following inequality is satisfied for all $(\mathbf{u}, \mathbf{y})$ in $S$ :

$$
\inf _{T \geq 0} \sum_{t=0}^{T} \gamma \rho(u(t))-\mu(y(t))>-\infty \text {. }
$$

In particular, when $\rho$ and $\mu$ are non-negative (and not identically zero), a notion of gain can be defined. Let $\rho: \mathcal{U} \rightarrow \mathbb{R}_{+}$and $\mu: \mathcal{Y} \rightarrow \mathbb{R}_{+}$be given non-negative functions. The $\rho / \mu$ gain of $S$ is the infimum of $\gamma$ such that (1) is satisfied.

A real valued function $d: \mathcal{A} \times \mathcal{A} \rightarrow \mathbb{R}$ is said to be symmetric if $d(a, b)=d(b, a)$ for all $a, b$ in $\mathcal{A}$ and positive definite if it satisfies:

1) $d(a, b) \geq 0, \forall a, b \in \mathcal{A}$;

2) $d(a, b)=0 \Leftrightarrow a=b$.

Definition 4: A system $S \subset \mathcal{U}^{\mathbb{Z}_{+}} \times \mathcal{Y}^{\mathbb{Z}_{+}}$is incrementally stable if there exists a finite non-negative constant $\gamma$ and a pair of symmetric positive definite functions $d_{\mathcal{U}}: \mathcal{U} \times \mathcal{U} \rightarrow \mathbb{R}_{+}$and $d_{\mathcal{Y}}: \mathcal{Y} \times \mathcal{Y} \rightarrow \mathbb{R}_{+}$such that the following inequality is satisfied for any $\left(\mathbf{u}_{1}, \mathbf{y}_{1}\right)$ and $\left(\mathbf{u}_{2}, \mathbf{y}_{2}\right)$ in $S$

$$
\inf _{T \geq 0} \sum_{t=0}^{T} \gamma d_{\mathcal{U}}\left(u_{1}(t), u_{2}(t)\right)-d_{\mathcal{Y}}\left(y_{1}(t), y_{2}(t)\right)>-\infty
$$

Given a particular choice of symmetric positive definite functions $d_{\mathcal{U}}$ and $d_{\mathcal{Y}}$, the infimum of $\gamma$ such that (2) is satisfied is called the $d_{\mathcal{U}} / d_{\mathcal{Y}}$ incremental gain of $S$.

Definition 5: A system $S \subset \mathcal{U}^{\mathbb{Z}_{+}} \times \mathcal{Y}^{\mathbb{Z}_{+}}$is externally unstable if there exists a finite constant $\tau \geq 0$ and $\left(\mathbf{u}, \mathbf{y}_{1}\right),\left(\mathbf{u}, \mathbf{y}_{2}\right)$ in $S$ such that $y_{1}\left(t^{\prime}\right) \neq y_{2}\left(t^{\prime}\right)$ for some $t^{\prime} \in[t, t+\tau]$, for all $t \geq 0$. $S$ is externally stable if it is not externally unstable.

\section{Stability of Interconnections}

The feedback interconnection $(S, \Delta) \subset \mathcal{U}^{\mathbb{Z}_{+}} \times \mathcal{Y}^{\mathbb{Z}_{+}}$of two systems $S \subset(\mathcal{U} \times \mathcal{W})^{\mathbb{Z}_{+}} \times(\mathcal{Y} \times \mathcal{Z})^{\mathbb{Z}_{+}}$and $\Delta \subset \mathcal{Z}^{\mathbb{Z}_{+}} \times \mathcal{W}^{\mathbb{Z}_{+}}$, as shown in Fig. 1, is the system:

$(S, \Delta)=\{(\mathbf{u}, \mathbf{y}) \mid \exists \mathbf{w}, \mathbf{z}$ such that

$$
((\mathbf{u}, \mathbf{w}),(\mathbf{y}, \mathbf{z})) \in S \text { and }(\mathbf{z}, \mathbf{w}) \in \Delta\} .
$$




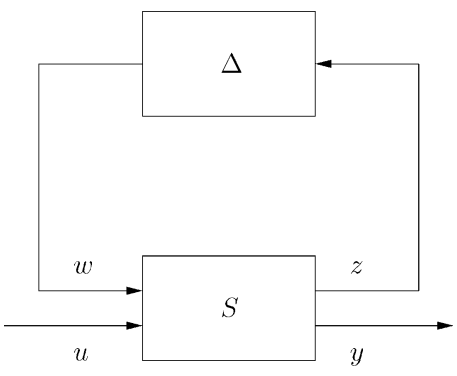

Fig. 1. Feedback interconnection of $S$ and $\Delta$.

Suppose that both $S$ and $\Delta$ are stable in the same sense (either gain stable or incrementally stable). The question is: What can be said about the stability of their feedback interconnection $(S, \Delta)$ ?

Theorem 1: (A Small Gain Theorem) Suppose that $S$ is $\rho_{S} / \mu_{S}$ gain stable and satisfies (1) with $\gamma=1$ for some $\rho_{S}: \mathcal{U} \times \mathcal{W} \rightarrow \mathbb{R}$ and $\mu_{S}: \mathcal{Y} \times \mathcal{Z} \rightarrow \mathbb{R}$, and that $\Delta$ is $\rho_{\Delta} / \mu_{\Delta}$ gain stable and satisfies (1) with $\gamma=1$ for some $\rho_{\Delta}: \mathcal{Z} \rightarrow \mathbb{R}$ and $\mu_{\Delta}: \mathcal{W} \rightarrow \mathbb{R}$. The interconnection $(S, \Delta)$ with input $u$ and output $y$ is $\rho / \mu$ gain stable for $\rho: \mathcal{U} \rightarrow \mathbb{R}$ and $\mu: \mathcal{Y} \rightarrow \mathbb{R}$ defined as

$$
\begin{aligned}
& \rho(u):=\sup _{w \in \mathcal{W}}\left\{\rho_{S}(u, w)-\mu_{\Delta}(w)\right\} \\
& \mu(y):=\inf _{z \in \mathcal{Z}}\left\{\mu_{S}(y, z)-\rho_{\Delta}(z)\right\}
\end{aligned}
$$

and satisfies (1) with $\gamma=1$.

Proof: By assumption, the feasible signals of $S$ satisfy

$$
\inf _{T \geq 0} \sum_{t=0}^{T} \rho_{S}(u(t), w(t))-\mu_{S}(y(t), z(t))>-\infty,
$$

and those of $\Delta$ satisfy

$$
\inf _{T \geq 0} \sum_{t=0}^{T} \rho_{\Delta}(z(t))-\mu_{\Delta}(w(t))>-\infty .
$$

For functions $\rho$ and $\mu$ defined in (3) and (4), (5) implies that all feasible signals of $S$ satisfy the following condition:

$$
\inf _{T \geq 0} \sum_{t=0}^{T} \rho(u(t))+\mu_{\Delta}(w(t))-\mu(y(t))-\rho_{\Delta}(z(t))>-\infty .
$$

Adding (6) to (7), and noting that the infimum of the sum of two functions is larger than or equal to the sum of the infimums of the functions, we get

$$
\inf _{T \geq 0} \sum_{t=0}^{T} \rho(u(t))-\mu(y(t))>-\infty .
$$

Hence, the interconnection $(S, \Delta)$ is $\rho / \mu$ gain stable and satisfies (1) with $\gamma=1$.

This formulation of the Small Gain Theorem is valid for arbitrary systems: In particular, when $S$ and $\Delta$ are systems over finite alphabets, "sup" and "inf" in (3) and (4) can be replaced by "max" and "min," respectively.

Theorem 2: (An Incremental Small Gain Theorem) Suppose that $S$ is incrementally stable with $d_{\mathcal{U}_{S}} / d_{\mathcal{Y}_{S}}$ incremental gain not exceeding 1 for some symmetric positive definite functions
$d_{\mathcal{U}_{S}}:(\mathcal{U} \times \mathcal{W}) \times(\mathcal{U} \times \mathcal{W}) \rightarrow \mathbb{R}_{+}$and $d_{\mathcal{Y}_{S}}:(\mathcal{Y} \times \mathcal{Z}) \times(\mathcal{Y} \times$ $\mathcal{Z}) \rightarrow \mathbb{R}_{+}$, and that $\Delta$ is incrementally stable with $d_{\mathcal{U}_{\Delta}} / d_{\mathcal{Y}_{\Delta}}$ incremental gain not exceeding 1 for some symmetric positive definite functions $d_{\mathcal{U}_{\Delta}}: \mathcal{Z} \times \mathcal{Z} \rightarrow \mathbb{R}_{+}$and $d_{\mathcal{Y}_{\Delta}}: \mathcal{W} \times \mathcal{W} \rightarrow$ $\mathbb{R}_{+}$. If functions $d_{\mathcal{U}}: \mathcal{U} \times \mathcal{U} \rightarrow \mathbb{R}$ and $d_{\mathcal{Y}}: \mathcal{Y} \times \mathcal{Y} \rightarrow \mathbb{R}$ defined as

$$
\begin{aligned}
& d_{\mathcal{U}}\left(u_{1}, u_{2}\right):= \\
& \sup _{w_{1}, w_{2} \in \mathcal{W}}\left\{d_{\mathcal{U}_{S}}\left(\left(u_{1}, w_{1}\right),\left(u_{2}, w_{2}\right)\right)-d_{\mathcal{Y}_{\Delta}}\left(w_{1}, w_{2}\right)\right\} \\
& d_{\mathcal{Y}}\left(y_{1}, y_{2}\right):= \\
& \inf _{z_{1}, z_{2} \in \mathcal{Z}}\left\{d_{\mathcal{Y}_{S}}\left(\left(y_{1}, z_{1}\right),\left(y_{2}, z_{2}\right)\right)-d_{\mathcal{U}_{\Delta}}\left(z_{1}, z_{2}\right)\right\}
\end{aligned}
$$

are positive-definite, the interconnection $(S, \Delta)$ with input $u$ and output $y$ is incrementally stable, and its $d_{\mathcal{U}} / d_{\mathcal{y}}$ incremental gain does not exceed 1.

Proof: Under the stated assumptions, it follows by an argument similar to that in the Proof of Theorem 1 that

$$
\inf _{T \geq 0} \sum_{t=0}^{T} d_{\mathcal{U}}\left(u_{1}(t), u_{2}(t)\right)-d_{\mathcal{Y}}\left(y_{1}(t), y_{2}(t)\right)>-\infty \text {. }
$$

If $d_{\mathcal{U}}$ and $d_{\mathcal{Y}}$, which are symmetric by definition, are positive definite, the interconnection $(S, \Delta)$ is incrementally stable and its $d_{\mathcal{U}} / d_{\mathcal{Y}}$ incremental gain does not exceed 1 .

\section{Comments on Stability of Systems and Interconnections}

It may be informative to note that Definition 3 can be equivalently re-written as: There exists a finite non-negative constant $\gamma$ such that for each $(\mathbf{u}, \mathbf{y}) \in S$ there exists a finite non-negative constant $C_{\mathbf{u}, \mathbf{y}}$ for which the following inequality holds for all $T \geq 0$ :

$$
\sum_{t=0}^{T} \mu(y(t)) \leq C_{\mathbf{u}, \mathbf{y}}+\gamma \sum_{t=0}^{T} \rho(u(t)) .
$$

Note that the proposed notion of gain stability is consistent with traditional notions of stability: For instance, a discrete-time LTI system that is internally stable (poles in the unit disk) is $\rho / \mu$ gain stable for functions $\rho$ and $\mu$ defined by $\rho(u)=\|u\|_{1}$ and $\mu(y)=\|y\|_{1}$. While less restrictive definitions of stability have been proposed for nonlinear systems, typically involving the use of gain functions (such as class $\mathcal{K}$ functions) [35], the use of such gain functions in Definition 3 is unlikely to offer any advantages over the use of a simple gain for systems over finite alphabets: This is due to the finiteness of the alphabet sets which ensures that the derivative of the gain function remains bounded at infinity if it is bounded elsewhere, and due to the possibility of appropriately rescaling functions $\rho$ and $\mu$. The relation between the proposed notion of gain stability and classical dissipation theory [36] should also be noted. In particular, a system that is dissipative with supply rate $\gamma \rho(u)-\mu(y)$ is $\rho / \mu$ gain stable. The choice of notation here, namely the use of "inf" and " $-\infty$ " rather than "sup" and " $+\infty$ " as is standard in Willems" framework, was made in keeping with the notation of IQC theory [18].

Definition 3 does not impose restrictions on the choice of functions $\rho$ and $\mu$. As such, given any system it is always possible to find some choice of $\rho$ and $\mu$ for which the system is $\rho / \mu$ gain stable: A judicious choice of functions is thus necessary to 
ensure that the corresponding gain stability condition describes a relevant property of the system. The case where $\rho$ and $\mu$ are zero on some $\mathcal{U}_{o} \subset \mathcal{U}$ and $\mathcal{Y}_{o} \subset \mathcal{Y}$, respectively, and positive elsewhere is of particular interest. The value of the gain in this case could, for instance, be indicative of the proportion of time in which the output deviates from the desired set $\mathcal{Y}_{o}$ to that in which the input deviates from the desired set $\mathcal{U}_{O}$ (see Example 3). Note that if the alphabet sets have some particular algebraic structure, there is a natural choice for $\mathcal{U}_{o}$ and $\mathcal{Y}_{o}$. For example, for an alphabet set with a group or field structure, the natural choice is the singleton consisting of the identity element of the group and the additive identity element of the field, respectively. In the traditional setting where the signals take their values in vector spaces over the reals, the natural choice of $\mathcal{U}_{o}$ and $\mathcal{Y}_{o}$ is the singleton consisting of the zero vector, with corresponding zero norm.

Remark 1: For a given system $S \subset \mathcal{U}^{\mathbb{Z}_{+}} \times \mathcal{Y}^{\mathbb{Z}_{+}}$over finite alphabets and a particular choice of $\mathcal{U}_{o} \subset \mathcal{U}$ and $\mathcal{Y}_{o} \subset \mathcal{Y}$, the existence of a finite $\gamma>0$ and functions $\rho: \mathcal{U} \rightarrow \mathbb{R}_{+}$and $\mu: \mathcal{Y} \rightarrow \mathbb{R}_{+}$, zero on $\mathcal{U}_{o}$ and $\mathcal{Y}_{o}$, respectively, and positive elsewhere, such that (1) is satisfied is an intrinsic property of the system (i.e., independent of the specific choice of $\rho$ and $\mu$ ); when such a $\gamma$ exists, the system is said to be gain stable about $\left(\mathcal{U}_{o}, \mathcal{Y}_{o}\right)$. Indeed, let $\rho_{1}: \mathcal{U} \rightarrow \mathbb{R}_{+}$and $\rho_{2}: \mathcal{U} \rightarrow \mathbb{R}_{+}$be zero on $\mathcal{U}_{o} \subset \mathcal{U}$ and positive elsewhere. Let $\mu_{1}: \mathcal{Y} \rightarrow \mathbb{R}_{+}$and $\mu_{2}: \mathcal{Y} \rightarrow \mathbb{R}_{+}$be zero on $\mathcal{Y}_{o} \subset \mathcal{Y}$ and positive elsewhere. Set

and

$$
c_{\rho}=\max _{u \in \mathcal{U}-\mathcal{U}_{o}} \frac{\rho_{1}(u)}{\rho_{2}(u)}
$$

$$
c_{\mu}=\min _{y \in \mathcal{Y}-\mathcal{Y}_{o}} \frac{\mu_{1}(y)}{\mu_{2}(y)}
$$

Note that $c_{\rho}$ and $c_{\mu}$ are finite when the alphabet sets are finite. For any non-negative constant $\gamma$ and any $T \geq 0$, we have:

$$
c_{\mu} \sum_{t=0}^{T} \gamma \frac{c_{\rho}}{c_{\mu}} \rho_{2}(u(t))-\mu_{2}(y(t)) \geq \sum_{t=0}^{T} \gamma \rho_{1}(u(t))-\mu_{1}(y(t)) .
$$

It follows that if there exists a non-negative constant $\gamma$, say $\gamma=$ $\gamma_{1}$, such that (1) holds for some choice of functions $\rho_{1}$ and $\mu_{1}$ zero on $\mathcal{U}_{o}$ and $\mathcal{Y}_{o}$ respectively and positive elsewhere; then for any other choice of functions $\rho_{2}$ and $\mu_{2}$ zero on $\mathcal{U}_{o}$ and $\mathcal{Y}_{o}$, respectively, and positive elsewhere, there exists a value $\gamma_{2} \geq 0$, in particular $\gamma_{2}=\gamma_{1} c_{\rho} / c_{\mu}$, for which (1) also holds for all $(\mathbf{u}, \mathbf{y}) \in S$.

The notion of incremental stability proposed in this paper captures the sensitivity of the output trajectories of a given system to perturbations in its input and initial conditions. Our motivation for defining this notion is to provide an alternative to gain stability when the latter may be too limited or too weak [37]-[40]. As should be expected, a discrete-time LTI system that is internally stable is incrementally stable in the sense of Definition 4 with $d_{\mathcal{U}}\left(u_{1}, u_{2}\right)=\left\|u_{1}-u_{2}\right\|_{p}$ and $d_{\mathcal{Y}}\left(y_{1}, y_{2}\right)=\left\|y_{1}-y_{2}\right\|_{p}$ where $\|\cdot\|_{p}$ denotes the standard $l_{p}$ norm for finite positive integer $p$.

Remark 2: By an argument similar to that made in Remark 1, it is clear that incremental stability (or lack of it) is an intrinsic property of a given system over finite alphabets. However, the

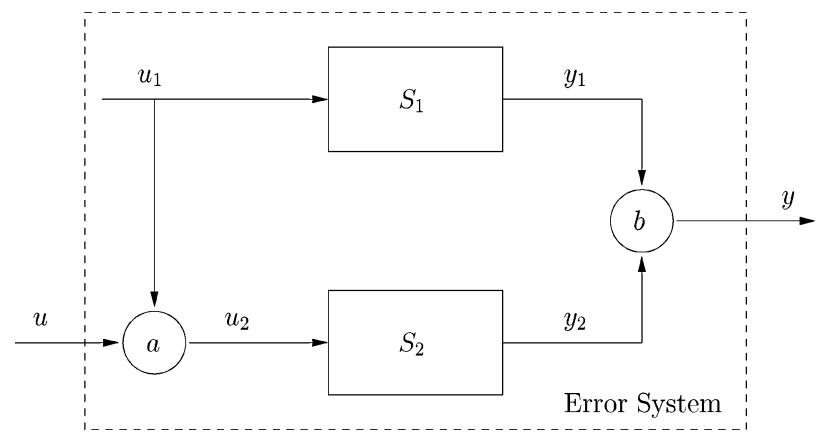

Fig. 2. The error system of $S_{1}$ and its approximation $S_{2}$.

numerical value of the incremental gain of a stable system depends on the choice of functions $d_{\mathcal{U}}$ and $d_{\mathcal{Y}}$.

The notion of external stability proposed in this paper captures the property that a system forgets its past, as evidenced by the evolution of its output trajectories. The nomenclature was chosen to emphasize the contrast between this notion and that of internal stability, concerned with the evolution of the state (internal) trajectories. Note that a system that is internally stable need not be externally stable: Example 5 in Section II-E illustrates this point.

Remark 3: For systems over finite alphabets, incremental stability is a stronger notion than external stability. Indeed, suppose that a system $S \subset \mathcal{U}^{\mathbb{Z}_{+}} \times \mathcal{Y}^{\mathbb{Z}_{+}}$is incrementally stable. Then for any pair of elements $\left(\mathbf{u}, \mathbf{y}_{1}\right)$ and $\left(\mathbf{u}, \mathbf{y}_{2}\right)$ in $S$, we have the following inequality:

$$
\sup _{T \geq 0} \sum_{t=0}^{T} d_{\mathcal{Y}}\left(y_{1}(t), y_{2}(t)\right)<\infty .
$$

When $\mathcal{Y}$ is finite, $d_{\mathcal{Y}}$ only takes on a finite number of values and the above inequality allows us to conclude that the system is externally stable. It will be shown in Section III-E that the two notions are equivalent for deterministic finite state machine systems.

The new formulation of the Small Gain Theorem proposed in this paper recovers the traditional Small Gain Theorem when applied in the traditional setting. Indeed, when gain stability of systems $S$ and $\Delta$ are interpreted as $l_{2}$ stability conditions, we have: $\rho_{S}(u, w)=\|u\|_{2}^{2}+\|w\|_{2}^{2}, \mu_{S}(y, z)=\|y\|_{2}^{2}+\|z\|_{2}^{2}$, $\mu_{\Delta}(w)=\|w\|_{2}^{2}, \rho_{\Delta}(z)=\|z\|_{2}^{2}$, and consequently $\rho(u)=$ $\sup _{w}\left(\|u\|_{2}^{2}+\|w\|_{2}^{2}-\|w\|_{2}^{2}\right)=\|u\|_{2}^{2}$ and $\mu(y)=\inf _{z}\left(\|y\|_{2}^{2}+\right.$ $\left.\|z\|_{2}^{2}-\|z\|_{2}^{2}\right)=\|y\|_{2}^{2}$. Our formulation thus recovers the standard small gain result: If each of $S$ and $\Delta$ are stable with $l_{2}$ gain not exceeding 1 , then so is their interconnection.

Remark 4: It follows from Theorem 1 that if two systems $S$ and $\Delta$ are $\rho_{S} / \mu_{S}$ and $\rho_{\Delta} / \mu_{\Delta}$ stable, respectively, satisfying (1) with $\gamma=1$, their interconnection $(S, \Delta)$ is $\rho / \mu$ gain stable and satisfies (1) with $\gamma=1$ for $\rho$ and $\mu$ given by

$$
\begin{aligned}
& \rho(u):=\sup _{w \in \mathcal{W}}\left\{\rho_{S}(u, w)-\tau \mu_{\Delta}(w)\right\} \\
& \mu(y):=\inf _{z \in \mathcal{Z}}\left\{\mu_{S}(y, z)-\tau \rho_{\Delta}(z)\right\}
\end{aligned}
$$

for any choice of scaling parameter $\tau>0$. Indeed, when the feasible signals of $\Delta$ satisfy (6), they also satisfy

$$
\inf _{T \geq 0} \sum_{t=0}^{T} \tau \rho_{\Delta}(z(t))-\tau \mu_{\Delta}(w(t))>-\infty
$$




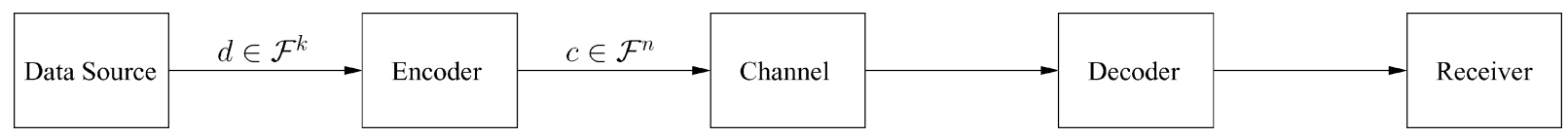

Fig. 3. Coding Setup.

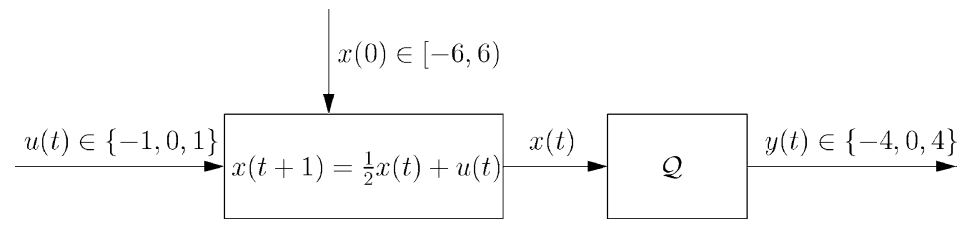

Fig. 4. Stable LTI system with state quantizer $Q$

for all $\tau>0$. The Proof of Theorem 1 thus follows through with " $\rho_{\Delta}$ " and " $\mu_{\Delta}$ " replaced by " $\tau \rho_{\Delta}$ " and " $\tau \mu_{\Delta}$ ", respectively. This scaling allows us to overcome some of the conservatism of the Small Gain Theorem.

In particular, we may be interested in proving stability of the interconnection $(S, \Delta)$ about $\left(\mathcal{U}_{o}, \mathcal{Y}_{o}\right)$ for some specific choice of $\mathcal{U}_{O} \subset \mathcal{U}$ and $\mathcal{Y}_{o} \subset \mathcal{Y}$. Theorem 1 allows us to verify this if $\rho$ and $\mu$ defined in (13) and (14) satisfy the requirement that they are zero on $\mathcal{U}_{o}$ and $\mathcal{Y}_{o}$, respectively, and positive elsewhere for some choice of $\tau>0$.

Remark 5: The Incremental Small Gain Theorem is similarly amenable to scaling thus easing some of its conservatism.

\section{E. Illustrative Examples}

The following simple examples illustrate each of the input/ output stability notions proposed in Section II-B.

Example 3: Let $S_{1}$ and $S_{2}$ be given systems over binary alphabets $\mathcal{U}_{1}=\mathcal{U}_{2}=\left\{\alpha_{1}, \alpha_{2}\right\}$ and $\mathcal{Y}_{1}=\mathcal{Y}_{2}=\left\{\beta_{1}, \beta_{2}\right\} . S_{2}$ is typically considered to be a good approximation of $S_{1}$ if it is a lower complexity system ${ }^{2}$ whose response to every input is close to that of $S_{1}$ to a similar input. This can be quantified by the $\rho / \mu$ gain of the error system (Fig. 2) with input $u \in\{0,1\}$ and output $y \in\{0,1\}$, where $\rho:\{0,1\} \rightarrow \mathbb{R}_{+}$ and $\mu:\{0,1\} \rightarrow \mathbb{R}_{+}$are simply the identity maps. Function $a:\left\{\alpha_{1}, \alpha_{2}\right\} \times\{0,1\} \rightarrow\left\{\alpha_{1}, \alpha_{2}\right\}$ is a "flip in input" transformation defined by

$a\left(u_{1}, u\right):= \begin{cases}\alpha_{1}, & \text { if }\left(u=0, u_{1}=\alpha_{1}\right) \text { or }\left(u=1, u_{1}=\alpha_{2}\right) \\ \alpha_{2}, & \text { if }\left(u=0, u_{1}=\alpha_{2}\right) \text { or }\left(u=1, u_{1}=\alpha_{1}\right)\end{cases}$

and function $b:\left\{\beta_{1}, \beta_{2}\right\} \times\left\{\beta_{1}, \beta_{2}\right\} \rightarrow\{0,1\}$ is a "binary comparator" defined by

$$
b\left(y_{1}, y_{2}\right):=\left\{\begin{array}{ll}
0, & \text { if } y_{1}=y_{2} \\
1, & \text { otherwise }
\end{array} .\right.
$$

In particular, note that when the error system is $\rho / \mu$ gain stable, the responses of $S_{1}$ and $S_{2}$ to the same input can only differ by a finite number of terms.

Example 4: Convolutional codes are widely used to add redundancy to data transmitted over noisy channels so as to en-

\footnotetext{
${ }^{2} \mathrm{~A}$ lower complexity system for our purposes is one that is simpler to analyze and to synthesize controllers for. The order of the state-space and the cardinality of the state set are adequate measures of system complexity for LTI and finite state systems, respectively.
}

able error free decoding at the receiver end (Fig. 3). A convolutional encoder is a map $E:\left(\mathcal{F}^{k}\right)^{\mathbb{Z}_{+}} \rightarrow\left(\mathcal{F}^{n}\right)^{\mathbb{Z}_{+}}$, where $\mathcal{F}$ is a finite field and $n$ and $k$ are integers with $n>k$, such that $\mathcal{C}=E\left(\left(\mathcal{F}^{k}\right)^{\mathbb{Z}_{+}}\right)$is a right shift-invariant linear subspace of $\left(\mathcal{F}^{n}\right)^{\mathbb{Z}_{+}}$. Given a convolutional code $\mathcal{C}$, the problem of finding an encoder for it can be formulated as the problem of finding a state-space realization for an invertible map $\phi_{E}: \mathcal{C} \rightarrow\left(\mathcal{F}^{k}\right)^{\mathbb{Z}_{+}}$. A good encoder is one that is "non-catastrophic", among other properties. An encoder is said to be catastrophic if two codewords $\mathbf{c}_{1}, \mathbf{c}_{2} \in \mathcal{C}$ differing by a finite number of terms correspond to two data sequences $\mathbf{d}_{1}, \mathbf{d}_{2} \in\left(\mathcal{F}^{k}\right)^{\mathbb{Z}}+$ differing by an infinite number of terms. Ensuring that the system over finite alphabets $S=\left\{(\mathbf{c}, \mathbf{d}) \in \mathcal{C} \times\left(\mathcal{F}^{k}\right)^{\mathbb{Z}}+\mid \mathbf{d}=\phi_{E}(\mathbf{c})\right\}$ is incrementally stable allows us to ensure that the corresponding encoder $\left(E=\phi_{E}^{-1}\right)$ is non-catastrophic.

Example 5: Consider a stable LTI system

$$
x(t+1)=\frac{1}{2} x(t)+u(t)
$$

whose input is restricted to three values, 0 and \pm 1 , and whose initial state is assumed to lie in the interval $[-6,6)$. Consider also an output quantizer $Q$, described (in the relevant range of values for input $x$ ) by

$$
y(t)= \begin{cases}-4, & -6 \leq x(t)<-2 \\ 0, & -2 \leq x(t)<2 \\ 4, & 2 \leq x(t)<6\end{cases}
$$

connected to the LTI system as shown in Fig. 4. Even though the LTI system is stable (pole inside the unit disk), the system with input $u$ and output $y$ is not externally stable: Consider a constant input $u(t)=1$ and two initial conditions $x_{1}(0)=0$ and $x_{2}(0)=4$. The corresponding constant outputs, $y_{1}(t)=0$ and $y_{2}(t)=4$, are unequal at every time step. The lack of external stability in a system has an important consequence [41]. We cannot expect to find an arbitrarily close approximation for such a system in the "traditional" sense of Example 3. A different approximation paradigm is needed for systems that are externally unstable.

\section{Stability of Deterministic Finite State Machine MODELS}

The remainder of the paper focuses on a special class of systems over finite alphabets where a specific process, modeled by 
a deterministic finite state machine (Definition 6), generates the feasible signals. This class of systems is interesting because it is expected to provide a set of tractable models from which, given a more complex system of interest, we can potentially choose a nominal model to approximate the complex one for the purpose of analysis and/or controller synthesis. The following questions are addressed in this section: Given a deterministic finite state machine $M$, how can we verify whether it is gain stable or incrementally stable? How can we compute its gain and incremental gain? Each of these questions is shown to reduce to verifying the existence of an appropriate storage function. Moreover, although incremental stability is generally stronger than external stability, the two notions are shown to be equivalent for systems modeled by finite state machines. It remains to be pointed out that the characterizations of stability in terms of energy storage descriptions of dissipativity are useful in the context of analysis, as described in this paper, and in the context of synthesis, in that they point to a characterization of an optimal control law [24].

\section{A. Deterministic Finite State Machine (DFM) Models}

Definition 6: A deterministic finite state machine (DFM) is a mathematical model described by a state transition equation (15) and an output equation (16):

$$
\begin{aligned}
& q(t+1)=f(q(t), u(t)) \\
& y(t)=g(q(t), u(t))
\end{aligned}
$$

where $t \in \mathbb{Z}_{+}, q(t) \in \mathcal{Q}, u(t) \in \mathcal{U}, y(t) \in \mathcal{Y}$, and where $\mathcal{Q}, \mathcal{U}$ and $\mathcal{Y}$ are finite sets.

$\mathcal{U}$ and $\mathcal{Y}$ are finite alphabet sets of possible instantaneous values of the input signal and the output signal, respectively. $\mathcal{Q}$ is the finite set of states of the DFM. $f: \mathcal{Q} \times \mathcal{U} \rightarrow \mathcal{Q}$ is the state transition function and $g: \mathcal{Q} \times \mathcal{U} \rightarrow \mathcal{Y}$ is the output function. The feasible signals set is given by:

$$
\begin{array}{r}
M=\left\{(\mathbf{u}, \mathbf{y}) \in \mathcal{U}^{\mathbb{Z}_{+}} \times \mathcal{Y}^{\mathbb{Z}_{+}} \mid \exists \mathbf{q} \in \mathcal{Q}^{\mathbb{Z}_{+}}\right. \\
\text {such that } \left.\mathbf{q}, \mathbf{u}, \mathbf{y} \text { satisfy }(15),(16), \forall t \in \mathbb{Z}_{+}\right\} .
\end{array}
$$

\section{B. Necessary and Sufficient Conditions for Gain Stability of DFM Models}

Characterizations of gain stability of a deterministic finite state machine in terms of energy storage descriptions of dissipativity properties are proposed in this section. The proofs of Theorems 3 and 4 are postponed until Section III-C.

Theorem 3: Consider a deterministic finite state machine $M$ defined by (15) and (16), and a function $\sigma: \mathcal{Q} \times \mathcal{U} \rightarrow \mathbb{R}$. The following two statements are equivalent:

a) for any $\mathbf{u} \in \mathcal{U}^{\mathbb{Z}}+$ and $q(0) \in \mathcal{Q}$, the following inequality is satisfied:

$$
\inf _{T \geq 0} \sum_{t=0}^{T} \sigma(q(t), u(t))>-\infty ;
$$

b) there exists a non-negative function $V: \mathcal{Q} \rightarrow \mathbb{R}_{+}$such that the inequality

$$
V(f(q, u))-V(q) \leq \sigma(q, u)
$$

holds for all $q \in \mathcal{Q}$ and $u \in \mathcal{U}$.
In particular, a choice of $\sigma(q, u)=\gamma \rho(u)-\mu(g(q, u))$ in Theorem 3 allows us to formulate a necessary and sufficient condition for $\rho / \mu$ gain stability of $M$.

Theorem 3 can be viewed as a variant of the standard result for discrete-time dissipative systems, specialized to the finite state setting. Indeed, the standard characterization of dissipativity is in terms of finiteness of the "available storage" function $S_{a}$ defined as

$$
S_{a}\left(q_{o}\right)=\sup _{T, u \in \mathcal{U}^{\mathbb{Z}_{T}}} \sum_{t=0}^{T}-\sigma(q(t), u(t)),
$$

with $q(0)=q_{o}$ [36], [42]. Note that, for an arbitrary system, finiteness of $S_{a}$ implies that statement (a) of Theorem 3 is satisfied; in our setting, finiteness of the state set ensures that finiteness of the available storage function is equivalent to statement (a). In the terminology of the theory of dissipative systems, $V$ in Theorem 3 is the storage function of the dissipative system $M$ with supply rate $\sigma$.

Given a deterministic finite state machine $M \subset \mathcal{U}^{\mathbb{Z}_{+}} \times \mathcal{Y}^{\mathbb{Z}_{+}}$ and $\mathcal{U}_{o} \subset \mathcal{U}, \mathcal{Y}_{o} \subset \mathcal{Y}$. It is possible to verify gain stability of $M$ about $\left(\mathcal{U}_{o}, \mathcal{Y}_{o}\right)$ by applying Theorem 3 to verify $\rho / \mu$ gain stability of $M$ for a particular choice of $\rho$ and $\mu$ zero on $\mathcal{U}_{o}$ and $\mathcal{Y}_{o}$, respectively, and positive elsewhere. However, it was noted earlier (see Remark 1 in Section II-D) that gain stability of a system about $\left(\mathcal{U}_{o}, \mathcal{Y}_{o}\right)$ is an intrinsic property of the system whenever the alphabet sets are finite. We thus expect to be able to formulate an alternative characterization of this property that does not involve a particular choice of $\rho$ and $\mu$. Theorem 4 presents such a characterization for deterministic finite state machines. The stability condition is again formulated as a dissipation inequality for the system with inputs restricted to values in $\mathcal{U}_{0}$.

Theorem 4: Consider a deterministic finite state machine $M$ defined by (15) and (16) and sets $\mathcal{U}_{o} \subset \mathcal{U}, \mathcal{Y}_{o} \subset \mathcal{Y} . M$ is gain stable about $\left(\mathcal{U}_{o}, \mathcal{Y}_{o}\right)$ if and only if there exists a non-negative function $V: \mathcal{Q} \rightarrow \mathbb{R}_{+}$such that the inequality

$$
V(f(q, u))-V(q) \leq-\mathbf{I}_{\mathcal{Y}-\mathcal{Y}_{o}}(g(q, u))
$$

holds for all $q \in \mathcal{Q}$ and $u \in \mathcal{U}_{o}$.

\section{Derivation of the Necessary and Sufficient Conditions for Gain Stability}

The following Lemma will be used in proving Theorem 3 .

Lemma 1: Consider a deterministic finite state machine $M$ defined by (15) and (16) and a function $\sigma: \mathcal{Q} \times \mathcal{U} \rightarrow \mathbb{R}$. Suppose that the following inequality is satisfied for any $q(0) \in \mathcal{Q}$ and $\mathbf{u} \in \mathcal{U}^{\mathbb{Z}_{+}}$:

$$
\inf _{T \geq 0} \sum_{t=0}^{T} \sigma(q(t), u(t))>-\infty .
$$

Then, for any $q(0) \in \mathcal{Q}, \mathbf{u} \in \mathcal{U}^{\mathbb{Z}_{T}}$ and $T \geq 0$, we have

$$
\sum_{t=0}^{T} \sigma(q(t), u(t)) \geq-n \sigma_{\max }
$$


where $n=\operatorname{card}(\mathcal{Q})$ and $\sigma_{\max }:=\max _{q, u}|\sigma(q, u)|$.

Proof: See Appendix I.

Remark 6: Note that finiteness of the set of states in a DFM model results in a stronger notion of gain stability, in the sense that a gain stable DFM admits a uniform upper bound for the parameters $C_{\mathbf{u}, \mathbf{y}}$ in (12). Indeed, it follows from Lemma 1 that $C=n \sigma_{\max }$ is one such uniform upper bound.

Proof: (Theorem 3) To prove that (b) $\Rightarrow$ (a), note that by summing up (19) along any trajectory from $t=0$ to $t=T$, we get

$$
\begin{aligned}
\sum_{t=0}^{T} \sigma(q(t), u(t)) & \geq V(f(q(T), u(T)))-V(q(0)) \\
& \geq \min _{q_{1}, q_{2}} V\left(q_{1}\right)-V\left(q_{2}\right),
\end{aligned}
$$

which implies (18).

To prove that (a) $\Rightarrow$ (b), define function $V: \mathcal{Q} \rightarrow \mathbb{R}$ as follows:

$$
V(q):=\sigma_{\max }+\sup _{T, \mathbf{u} \in \mathcal{U}^{\mathbb{Z}_{T}}} \sum_{t=0}^{T}-\sigma(q(t), u(t))
$$

where $\sigma_{\max }=\max _{q, u}|\sigma(q, u)|$ and $\mathbf{q}$ is the state trajectory associated with initial condition $q$ and input $\mathbf{u}$. It follows from Lemma 1 that the right hand side of (23) is bounded above and hence $V$ is well defined. Moreover, $V$ is non-negative by construction. Finally, we have

$$
\begin{aligned}
V(f(q, u))-V(q) \\
=\sup _{T, \mathbf{u} \in \mathcal{U}^{\mathbb{Z}_{T}}} \sum_{t=0}^{T}-\sigma(\hat{q}(t), u(t)) \\
\quad-\sup _{T, \mathbf{u} \in \mathcal{U}^{\mathbb{Z}_{T}}} \sum_{t=0}^{T}-\sigma((q(t), u(t))) \\
\leq \sup _{T, \mathbf{u} \in \mathcal{U}^{\mathbb{Z}_{T}}} \sum_{t=0}^{T}-\sigma(\hat{q}(t), u(t)) \\
\quad+\left(\sigma(q, u)-\sup _{T, \mathbf{u} \in \mathcal{U}^{\mathbb{Z}_{T-1}}} \sum_{t=1}^{T}-\sigma(q(t), u(t))\right) \\
=\sup _{T, \mathbf{u} \in \mathcal{U}^{\mathbb{Z}_{T}}} \sum_{t=0}^{T}-\sigma(\hat{q}(t), u(t)) \\
\quad+\left(\sigma(q, u)-\sup _{T-1, \mathbf{u} \in \mathcal{U}^{\mathbb{Z}_{T-1}}} \sum_{t=0}^{T-1}-\sigma(\hat{q}(t), u(t))\right) \\
=\sigma(q, u)
\end{aligned}
$$

where $q(0)=q$ and $q(1)=\hat{q}(0)=f(q, u)$.

Lemmas 2 and 3 will be additionally needed to prove Theorem 4.

Lemma 2: Consider a deterministic finite state machine $M$ defined by (15) and (16) and a function $\sigma: \mathcal{Q} \times \mathcal{U} \rightarrow \mathbb{R}$. If there exists a function $V: \mathcal{Q} \rightarrow \mathbb{R}$ such that for all $q \in \mathcal{Q}$ and $u \in \mathcal{U}$

$$
V(f(q, u))-V(q) \leq \sigma(q, u)
$$

then for any $\tau>0, \mathbf{u} \in \mathcal{U}^{\mathbb{Z}_{\tau-1}}$ and corresponding $\mathbf{q} \in \mathcal{Q}^{\mathbb{Z}_{\tau}}$ satisfying $q(0)=q(\tau)$, we have:

$$
\sum_{t=0}^{\tau-1} \sigma(q(t), u(t)) \geq 0
$$

Proof: By summing up (24) along any trajectory from $t=$ 0 to $t=T$, we get

$$
\begin{aligned}
\sum_{t=0}^{T} \sigma(q(t), u(t)) & \geq V(f(q(T), u(T)))-V(q(0)) \\
& \geq \min _{q_{1}, q_{2}} V\left(q_{1}\right)-V\left(q_{2}\right) .
\end{aligned}
$$

Suppose there exists a $\tau>0, u \in \mathcal{U}^{\mathbb{Z}_{\tau-1}}$ and corresponding $\mathbf{q} \in \mathcal{Q}^{\mathbb{Z}_{\tau}}$, with $q(0)=q(\tau)$, such that $\sum_{t=0}^{\tau-1} \sigma(q(t), u(t))<0$. We can construct a periodic input such that, for initial condition $q(0)$, the summation $\sum_{t=0}^{T} \sigma(q(t), u(t))$ can be made arbitrarily negative for large enough $T$, thus violating (25) and leading to a contradiction.

Lemma 3: Consider a deterministic finite state machine $M$ defined by (15) and (16) and sets $\mathcal{U}_{o} \subset \mathcal{U}, \mathcal{Y}_{o} \subset \mathcal{Y}$. If there exists a function $V: \mathcal{Q} \rightarrow \mathbb{R}$ such that for all $q \in \mathcal{Q}$ and $u \in \mathcal{U}_{o}$,

$$
V(f(q, u))-V(q) \leq-\mathbf{I}_{\mathcal{Y}-\mathcal{Y}_{o}}(g(q, u))
$$

then for any $\tau>0, \mathbf{u} \in \mathcal{U}^{\mathbb{Z}_{\tau-1}}$ and corresponding $\mathbf{q} \in \mathcal{Q}^{\mathbb{Z} \tau}$ with $q(0)=q(\tau)$, the inequality

$$
\sum_{t=0}^{\tau-1} \gamma \mathbf{I}_{\mathcal{U}-\mathcal{U}_{o}}(u(t))-\mathbf{I}_{\mathcal{Y}-\mathcal{Y}_{o}}(g(q(t), u(t))) \geq 0
$$

holds for $\gamma=\operatorname{card}(\mathcal{Q})$.

Proof: See Appendix II.

We are now ready to prove Theorem 4 .

Proof: (Theorem 4) To prove sufficiency, we will show that for any $\mathbf{u} \in \mathcal{U}^{\mathbb{Z}+}$ and $q \in \mathcal{Q}$, the following inequality is satisfied for all $T \geq 0$ :

$$
\sum_{t=0}^{T} \gamma \mathbf{I}_{\mathcal{U}-\mathcal{U}_{o}}(u(t))-\mathbf{I}_{\mathcal{Y}-\mathcal{Y}_{o}}(g(q(t), u(t))) \geq-C
$$

with $\gamma=\operatorname{card}(\mathcal{Q})$ and $C=\operatorname{card}(\mathcal{Q}) \operatorname{card}(\mathcal{U})$. This is clearly the case when $T \leq C$. If $T>C$, there must exist two integers $t^{\prime}$ and $t^{\prime \prime}$ in $\mathbb{Z}_{T}$, with $t^{\prime}<t^{\prime \prime}$, such that $q\left(t^{\prime}\right)=q\left(t^{\prime \prime}\right)$. Thus, it follows from Lemma 3 that we can construct new state and input sequences, $\overline{\mathbf{q}} \in \mathcal{Q}^{\mathbb{Z}_{T^{\prime}}}$ and $\overline{\mathbf{u}} \in \mathcal{U}^{\mathbb{Z}_{T^{\prime}}}$, where $T^{\prime}=T-\left(t^{\prime \prime}-t^{\prime}\right)$, such that

$$
\begin{aligned}
& \sum_{t=0}^{T^{\prime}} \gamma \mathbf{I}_{\mathcal{U}-\mathcal{U}_{o}}(\bar{u}(t))-\mathbf{I}_{\mathcal{Y}-\mathcal{Y}_{o}}(g(\bar{q}(t), \bar{u}(t))) \geq-C \\
& \Rightarrow \sum_{t=0}^{T} \gamma \mathbf{I}_{\mathcal{U}-\mathcal{U}_{o}}(u(t))-\mathbf{I}_{\mathcal{Y}-\mathcal{Y}_{o}}(g(q(t), u(t))) \geq-C .
\end{aligned}
$$

Now, for any $T_{0}>C$, by the preceding argument, we can construct a finite sequence of integers $T_{0}>\cdots>C \geq T_{k_{o}}$ 
and corresponding state and input sequences $\mathbf{q}_{k} \in \mathcal{Q}^{\mathbb{Z}_{T_{k}}}$ and $\mathbf{u}_{k} \in \mathcal{U}^{\mathbb{Z}_{T_{k}}}$ such that

$$
\begin{array}{r}
\sum_{t=0}^{T_{k+1}} \gamma \mathbf{I}_{\mathcal{U}-\mathcal{U}_{o}}\left(u_{k+1}(t)\right)-\mathbf{I}_{\mathcal{Y}-\mathcal{Y}_{o}}\left(g\left(q_{k+1}(t), u_{k+1}(t)\right)\right) \geq-C \\
\Rightarrow \sum_{t=0}^{T_{k}} \gamma \mathbf{I}_{\mathcal{U}-\mathcal{U}_{o}}\left(u_{k}(t)\right)-\mathbf{I}_{\mathcal{Y}-\mathcal{Y}_{o}}\left(g\left(q_{k}(t), u_{k}(t)\right)\right) \geq-C .
\end{array}
$$

Hence, it follows that (28) is satisfied for all $T \geq 0$.

To prove necessity, suppose that $M$ is gain stable about $\left(\mathcal{U}_{o}, \mathcal{Y}_{o}\right)$. Define function $V: \mathcal{Q} \rightarrow \mathbb{R}_{+}$as follows:

$$
V(q):=\sup _{T, \mathbf{u} \in \mathcal{U}_{o}^{\mathbb{Z}_{T}}} \sum_{t=0}^{T} \mathbf{I}_{\mathcal{Y}}-\mathcal{Y}_{o}(g(q(t), u(t)))
$$

where $\mathbf{q}$ is the state trajectory corresponding to initial state $q$ and input $\mathbf{u} \in \mathcal{U}_{o}^{\mathbb{Z}_{+}} . V$ is non-negative by construction, being the supremum of a set containing $0 . V$ is also finite: Indeed, application of Lemma 1 with

$$
\sigma(q, u)=\gamma \mathbf{I}_{\mathcal{U}-\mathcal{U}_{o}}(u)-\mathbf{I}_{\mathcal{Y}-\mathcal{Y}_{o}}(g(q, u))
$$

implies the following inequality for all $\mathbf{u} \in \mathcal{U}^{\mathbb{Z}_{T}}, T \geq 0$ :

$$
\sum_{t=0}^{T} \mathbf{I}_{\mathcal{Y}-\mathcal{Y}_{o}}(g(q(t), u(t))) \leq n \sigma_{\max }+\sum_{t=0}^{T} \gamma I_{\mathcal{U}-\mathcal{U}_{o}}(u(t)) .
$$

In particular, when $\mathbf{u} \in \mathcal{U}_{o}^{\mathbb{Z}_{T}}$, the summation on the right hand side vanishes and we get a constant upper bound for the summation on the left, which establishes finiteness of $V$. Finally, it follows from the definition that

$$
\begin{aligned}
V(f(q, u))-V(q)= & \sup _{T, \mathbf{u} \in \mathcal{U}_{o}^{\mathbb{Z}_{T}}} \sum_{t=0}^{T} \mathbf{I}_{\mathcal{Y}-\mathcal{Y}_{o}}(g(\hat{q}(t), u(t))) \\
& -\sup _{T, \mathbf{u} \in \mathcal{U}_{o}^{\mathbb{Z}} T} \sum_{t=0}^{T} \mathbf{I}_{\mathcal{Y}} \mathcal{Y}_{o}(g(q(t), u(t)))
\end{aligned}
$$

where $q(0)=q$ and $\hat{q}(0)=f(q, u)$. Moreover, we have

$$
\begin{aligned}
& \sup _{T, \mathbf{u} \in \mathcal{U}_{o}^{\mathbb{Z}_{T}}} \sum_{t=0}^{T} \mathbf{I}_{\mathcal{Y}-\mathcal{Y}_{o}}(g(q(t), u(t))) \\
& \geq \mathbf{I}_{\mathcal{Y}-\mathcal{Y}_{o}}(g(q, u))+\sup _{T, \mathbf{u} \in \mathcal{U}_{o}^{\mathbb{Z}_{T-1}}} \sum_{t=1}^{T} \mathbf{I}_{\mathcal{Y}-\mathcal{Y}_{o}}(g(q(t), u(t))) \\
& =\mathbf{I}_{\mathcal{Y}-\mathcal{Y}_{o}}(g(q, u)) \\
& \quad+\sup _{T-1, \mathbf{u} \in \mathcal{U}_{o}^{\mathbb{Z}}} \sum_{t=0}^{T-1} \mathbf{I}_{\mathcal{Y}-\mathcal{Y}_{o}}(g(\hat{q}(t), u(t)))
\end{aligned}
$$

where $q(1)=\hat{q}(0)=f(q, u)$. Hence (20) holds for every $q \in$ $\mathcal{Q}, u \in \mathcal{U}_{o}$.

\section{Incremental Stability of DFM Models}

Necessary and sufficient conditions for incremental stability and validity of an incremental gain bound of a deterministic finite state machine $M$ can be readily derived from Theorems 4 and 3, respectively, in Section III-B. The basic idea is that incremental stability of $M$ can be equivalently described in terms of gain stability of a new system $\hat{M}$ (referred to as a "supersystem" in [40]) consisting of two copies of $M$ driven in parallel by two different inputs and initial conditions. Indeed, let $M$ be a deterministic finite state machine defined by (15) and (16) and consider $\hat{M}$ with input and output alphabets $\hat{\mathcal{U}}=\mathcal{U} \times \mathcal{U}$ and $\hat{\mathcal{Y}}=\mathcal{Y} \times \mathcal{Y}$, state set $\hat{\mathcal{Q}}=\mathcal{Q} \times \mathcal{Q}$, state transition function $\hat{f}: \hat{\mathcal{Q}} \times \hat{\mathcal{U}} \rightarrow \hat{\mathcal{Q}}$ defined by

$$
\hat{f}(q, u):=\left(f\left(q_{1}, u_{1}\right), f\left(q_{2}, u_{2}\right)\right)
$$

and output function $\hat{g}: \hat{\mathcal{Q}} \times \hat{\mathcal{U}} \rightarrow \hat{\mathcal{Y}}$ defined by

$$
\hat{g}(q, u):=\left(g\left(q_{1}, u_{1}\right), g\left(q_{2}, u_{2}\right)\right) .
$$

Thus, $\hat{M}$ is described by the following state transition (31) and output (32) equations:

$$
\begin{aligned}
& q(t+1)=\hat{f}(q(t), u(t)) \\
& y(t+1)=\hat{g}(q(t), u(t)) .
\end{aligned}
$$

The following statements hold for $M$ and the corresponding $\hat{M}$ constructed as described earlier.

Lemma 4: $(\mathbf{u}, \mathbf{y}) \in \hat{M}$ iff $\left(\mathbf{u}_{1}, \mathbf{y}_{1}\right),\left(\mathbf{u}_{2}, \mathbf{y}_{2}\right) \in M$.

Proof:

$$
\begin{aligned}
(\mathbf{u}, \mathbf{y}) \in \hat{M} \Leftrightarrow & \exists \mathbf{q}=\left(\mathbf{q}_{1}, \mathbf{q}_{2}\right) \text { such that } \mathbf{q}, \mathbf{u}, \mathbf{y} \\
& \text { satisfy (31) and (32), } \forall t \in \mathbb{Z}_{+} \\
\Leftrightarrow & \exists \mathbf{q}_{1}, \mathbf{q}_{2} \text { such that } \mathbf{q}_{1}, \mathbf{u}_{1}, \mathbf{y}_{1} \text { and } \\
& \quad \mathbf{q}_{2}, \mathbf{u}_{2}, \mathbf{y}_{2} \text { satisfy }(15) \text { and }(16), \forall t \in \mathbf{Z}_{+} \\
\Leftrightarrow & \left(\mathbf{u}_{1}, \mathbf{y}_{1}\right),\left(\mathbf{u}_{2}, \mathbf{y}_{2}\right) \in M .
\end{aligned}
$$

Theorem 5: Let $\hat{\mathcal{U}}_{o}=\{(u, u) \mid u \in \mathcal{U}\}$ and $\hat{\mathcal{Y}}_{o}=\{(y, y) \mid y \in$ $\mathcal{Y}\} . M$ is incrementally stable iff $\hat{M}$ is gain stable about $\left(\hat{\mathcal{U}}_{0}, \hat{\mathcal{Y}}_{o}\right)$.

Proof: $M$ is incrementally stable

$\Leftrightarrow \exists \gamma \geq 0$, symmetric positive-definite functions $\rho: \mathcal{U} \times \mathcal{U} \rightarrow \mathbb{R}_{+}, \mu: \mathcal{Y} \times \mathcal{Y} \rightarrow \mathbb{R}_{+}$such that any two pairs $\left(\mathbf{u}_{1}, \mathbf{y}_{1}\right),\left(\mathbf{u}_{2}, \mathbf{y}_{2}\right)$ in $M$ satisfy

$$
\inf _{T \geq 0} \sum_{t=0}^{T} \gamma \rho\left(u_{1}(t), u_{2}(t)\right)-\mu\left(y_{1}(t), y_{2}(t)\right)>-\infty
$$

$\Leftrightarrow \exists \gamma \geq 0$ and symmetric functions $\rho: \mathcal{U} \times \mathcal{U} \rightarrow \mathbb{R}_{+}$and $\mu: \mathcal{Y} \times \mathcal{Y} \rightarrow \mathbb{R}_{+}$zero on $\hat{\mathcal{U}}_{o}$ and $\hat{\mathcal{Y}}_{o}$, respectively, and positive elsewhere such that any feasible signal $(\mathbf{u}, \mathbf{y})$ in $\hat{M}$ satisfies:

$$
\inf _{T \geq 0} \sum_{t=0}^{T} \gamma \rho(u(t))-\mu(y(t))>-\infty
$$

$\Leftrightarrow \hat{M}$ is gain stable about $\left(\hat{\mathcal{U}}_{o}, \hat{\mathcal{Y}}_{o}\right)$.

(The second equivalence follows from Lemma 4).

\section{E. Relating Incremental and External Stability}

While incremental stability is generally stronger than external stability, it will be shown next that the two notions are equivalent for DFM models.

Theorem 6: A deterministic finite state machine $M$ defined by (15) and (16) is externally stable iff it is incrementally stable. 
Proof: Sufficiency was shown in Remark 3 in Section II-D. To prove necessity, suppose that $M$ is externally stable and consider $V: \mathcal{Q} \times \mathcal{Q} \rightarrow \mathbb{R}_{+}$defined as

$$
V\left(q_{1}, q_{2}\right):=\sup _{T, u \in \mathcal{U}^{\mathbb{Z}_{T}}} \sum_{t=0}^{T} \phi\left(q_{1}(t), q_{2}(t), u(t)\right)
$$

where $\phi: \mathcal{Q} \times \mathcal{Q} \times \mathcal{U} \rightarrow\{0,1\}$ is defined by

$$
\phi\left(q_{1}, q_{2}, u\right):= \begin{cases}0, & g\left(q_{1}, u\right)=g\left(q_{2}, u\right) \\ 1, & \text { otherwise }\end{cases}
$$

where $\mathbf{q}_{1}$ and $\mathbf{q}_{2}$ are the state trajectories corresponding to initial states $q_{1}$ and $q_{2}$, respectively, and input $\mathbf{u}$. It follows from the definition of $V$, using an argument similar to the one used in the proofs of Theorems 3 and 4, that the following inequality holds for any $u \in \mathcal{U}$ :

$$
V\left(q_{1}, q_{2}\right) \geq \phi\left(q_{1}, q_{2}, u\right)+V\left(f\left(q_{1}, u\right), f\left(q_{2}, u\right)\right) .
$$

What is left to show is that $V$ is bounded. We claim that $V \leq$ $a n^{2}$, where $a=\operatorname{card}(\mathcal{U})$ and $n=\operatorname{card}(\mathcal{Q})$. The proof is by contradiction: Suppose that $V$ in unbounded; then there exists a pair of initial conditions $q_{1}, q_{2}$, a positive integer $T$, and an input sequence $\mathbf{u} \in \mathcal{U}^{\mathbb{Z}_{T}}$ with corresponding state sequences $\mathbf{q}_{1}, \mathbf{q}_{2} \in \mathcal{Q}^{\mathbb{Z}_{T}}$, such that $q_{1}=q_{1}(0)=q_{1}(T), q_{2}=q_{2}(0)=$ $q_{2}(T), u(0)=u(T)$ and $\phi\left(q_{1}, q_{2}, u(0)\right)=1$. We can then construct two periodic feasible signals $\left(\mathbf{u}_{o}, \mathbf{y}_{1}\right)$ and $\left(\mathbf{u}_{o}, \mathbf{y}_{2}\right)$, corresponding to initial conditions $q_{1}$ and $q_{2}$ and periodic input $\mathbf{u}_{o}$ defined as $u_{o}(k T+l):=u(l)$ for $k \in \mathbb{Z}_{+}$and $l \in\{0, \ldots, T-1\}$, which violate the condition in Definition 5, hence contradicting our assumption of external stability. Since $V$ is finite, non-negative and satisfies (33), it follows from Theorems 4 and 5 that $M$ is incrementally stable.

\section{F. A Note About the Search for Storage Functions}

It follows from Sections III-B and III-D that verifying gain and incremental stability of a DFM, as well as verifying a particular gain or incremental gain bound, can be done by checking feasibility of a linear program of the form

$$
A x \geq b
$$

where the decision variable $x$ is the vector of values of the storage function $V$. Similarly, computing a particular gain (when well defined) or incremental gain of a DFM can be done by solving a linear program of the form

$$
\begin{array}{rc}
\min & c^{\prime} x \\
\text { subject to } & A x \geq b
\end{array}
$$

where the decision variable $x$ is the vector of values of the (incremental) gain and the corresponding storage function $V$. Note that it is not necessary to enforce $x \geq 0$ in the above linear programs since a feasible non-negative solution exists whenever a feasible solution exists. The following simple example demonstrates this procedure.

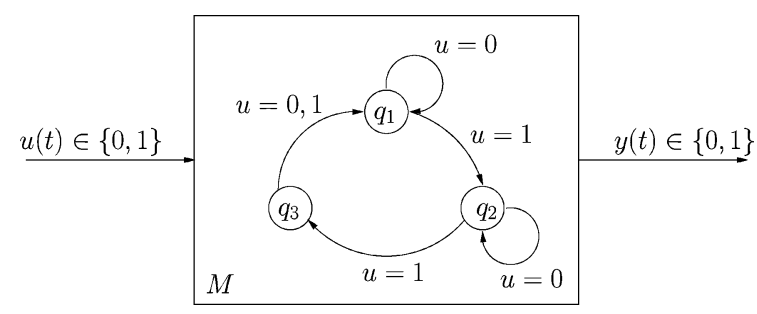

Fig. 5. The DFM in Example 6.

Example 6: Consider a DFM $M$ with three states $q_{1}, q_{2}$, and $q_{3}$, binary input and output alphabets $\mathcal{U}=\mathcal{Y}=\{0,1\}$, output function $g$ defined by

$$
g\left(q_{i}, u\right)= \begin{cases}u, & \text { for } i \in\{1,2\} \\ 0, & \text { for } i=3\end{cases}
$$

and state transitions as shown in Fig. 5. Let $\rho:\{0,1\} \rightarrow \mathbb{R}_{+}$, $\mu:\{0,1\} \rightarrow \mathbb{R}_{+}$be the identity maps. $M$ is $\rho / \mu$ gain stable with gain 1 . Stability can be verified using Theorem 4 (with $\left.\mathcal{U}_{o}=\mathcal{Y}_{o}=\{0\}\right)$ by finding a feasible solution $(V=0)$ to the linear program:

$$
\begin{aligned}
& V_{1}-V_{1} \leq 0 \\
& V_{2}-V_{2} \leq 0 \\
& V_{1}-V_{3} \leq 0
\end{aligned}
$$

with $V_{i}$ being shorthand notation for $V\left(q_{i}\right)$. The $\rho / \mu$ gain of $M$ can be explicitly computed using Theorem 3 by finding the optimal solution $(\gamma=1)$ of the linear program:

$$
\begin{aligned}
& \min \gamma \\
& \text { subject to } V_{1}-V_{1} \leq 0 \\
& V_{2}-V_{1}-\gamma \leq-1 \\
& V_{2}-V_{2} \leq 0 \\
& V_{3}-V_{2}-\gamma \leq-1 \\
& V_{1}-V_{3} \leq 0 \\
& V_{1}-V_{3}-\gamma \leq 0
\end{aligned}
$$

While an off the shelf LP solver can in principle be used in this setting, it is not advisable for two reasons. On one hand, the linear programs in question are highly structured: Matrix $A$ is sparse, with integer entries taking one of three values $(-1,0$, or 1 ), with at most three non-zero entries per row, and with a row sum of either -1 or 0 . Moreover, in the linear programs associated with verifying stability or incremental stability, vector $b$ consists of integer entries taking the values 0 or -1 . Finally, in the linear programs associated with gain computation, vector $c$ is an all zero vector except for a single unity entry. On the other hand, the DFM models of interest, typically being approximate models of potentially complex dynamical systems, are expected to have a large number of states. In view of this structure and the potential size of the problems of interest, it is important to develop specialized algorithms with better worst-case complexity 
bounds and better practical performance than generic LP solution algorithms. An approach for addressing this issue is presented in the next section. Alternative approaches involving the development and use of specialized LP solvers are also potentially possible.

\section{A COMPUTATIONAL ALGORITHM FOR STABILITY AND GAIN VERIFICATION}

It is shown in this section that the problem of verifying a dissipation inequality for a deterministic finite state machine is equivalent to the problem of verifying the non-existence of negative cost cycles in an appropriately constructed network. As such, verification of gain stability, incremental stability and particular gain bounds can be carried out efficiently by adapting solution techniques for discrete shortest path problems. We begin in Section IV-A with a brief review of relevant network flow concepts. The construction of the underlying network flow problem is described in Section IV-B, where the relation between DFM stability analysis and the network flow problem is established. In Section IV-C, a computational solution algorithm, adapted from the Bellman-Ford algorithm [43], is proposed and shown to remain strongly polynomial, with worst-case complexity $\mathcal{O}\left(n^{2}\right)$ for a network with $n$ nodes and at most $a n$ edges, where $a \ll n$.

\section{A. A Brief Review of Network Flows}

A directed graph $G=(\mathcal{N}, \mathcal{E})$ is a set of nodes $\mathcal{N}=\{1, \ldots, n\}$ and a set of directed edges $\mathcal{E} \subset \mathcal{N}^{2}$, where $(i, j) \in \mathcal{E}$ is an edge outgoing from node $i$ and incoming into node $j$. A network $G=(\mathcal{N}, \mathcal{E}, c)$ is a directed graph with additional numerical information associated with its edges, in particular a cost function $c: \mathcal{E} \rightarrow \mathbb{R}$. The shorthand notation $c_{i j}$ is used for $c(i, j)$. A path is a sequence of edges $\left(i_{1}, i_{2}\right),\left(i_{2}, i_{3}\right), \ldots,\left(i_{k-1}, i_{k}\right)$; a path is simple if $i_{1}, \ldots, i_{k}$ are all distinct. A cycle is a path in which $i_{1}=i_{k}$; a cycle is simple if $i_{1}, \ldots, i_{k-1}$ are distinct. The cost of a path is the sum of the costs of its edges.

Consider a network $G=(\mathcal{N}, \mathcal{E}, c)$. The all-to-one discrete shortest path problem can be stated as follows: Given a choice of destination node (say node $k$ ), find the shortest (i.e., least costly) directed path from each of the remaining nodes to $k$. A class of algorithms, collectively referred to as label-correcting algorithms, solve this problem. The basic idea is to associate with each node $j$ a distance label $d(j)$, which provides an upper bound on the length of the shortest path from node $j$ to the destination node while the algorithm is running. While various implementations of label-correcting algorithms exist (see [44] for a detailed discussion), they all terminate either when the lengths of all the shortest paths have been computed or when a negative cost cycle has been discovered. The following well-known optimality condition (refer to [44] for a proof) allows us to assess whether a given set of distance labels represents the shortest path lengths to the destination node.

Lemma 5: Consider a network $G=(\mathcal{N}, \mathcal{E}, c)$ with fixed edge costs $c_{i j}$, and consider a function $d: \mathcal{N} \rightarrow \mathbb{R}$ with $d(k)=0$, where node $k$ is the destination node. Suppose that there are no outgoing edges from $k$, and that for every $j \in \mathcal{N}-\{k\}$, $d(j)$ denotes the length of a directed path from node $j$ to node $k$. Function $d$ defines the shortest path lengths iff the following condition is satisfied:

$$
d(i) \leq d(j)+c_{i j}, \forall(i, j) \in \mathcal{E} \text {. }
$$

\section{B. A Related Network Problem}

Let $M$ be a deterministic finite state machine defined by (15) and (16) and consider a dissipation inequality as in (19), repeated here for convenience

$$
V(f(q, u))-V(q) \leq \sigma(q, u), \text { for all } q \in \mathcal{Q}, u \in \mathcal{U}
$$

Let $n=\operatorname{card}(\mathcal{Q})$. We can associate with $M$ and the given dissipation inequality a network $G_{M}$ constructed as follows:

$$
\begin{aligned}
\mathcal{N} & :=\{1, \ldots, n\} \\
\mathcal{E} & :=\left\{(i, j) \in \mathcal{N} \times \mathcal{N} \mid \exists u \in \mathcal{U} \text { such that } q_{j}=f\left(q_{i}, u\right)\right\} \\
c_{i j} & :=\min _{u \mid f\left(q_{i}, u\right)=q_{j}} \sigma\left(q_{i}, u\right) .
\end{aligned}
$$

Lemma 6: $M$ satisfies inequality (19) iff $G_{M}$ has no negative cost cycles.

Proof: Necessity follows from Lemma 2. To prove sufficiency, suppose that $G_{M}$ has no negative cost cycles. Let $\mathcal{P}(i)$ be the set of all paths starting at node $i$, and let $c_{p}$ be the cost of path $p \in \mathcal{P}(i)$. Define a function $\bar{V}: \mathcal{N} \rightarrow \mathbb{R}$ by the following rule: $\bar{V}(i):=\sup _{p \in \mathcal{P}(i)}\left\{-c_{p}\right\}$. Let $\mathcal{P}^{\prime}(i)$ denote the subset of simple paths starting at node $i$. Note that $\bar{V}(i)=$ $\sup _{p \in \mathcal{P}^{\prime}(i)}\left\{-c_{p}\right\}$, since it is not possible to decrease the length of a path by adding cycles to it. Thus $\bar{V}$ is bounded by construction (the cost of each edge is finite and the maximum number of edges in any simple path is $n-1)$. Moreover, $\bar{V}(i) \geq-c_{i j}+$ $\bar{V}(j), \forall(i, j) \in \mathcal{E}$. Thus, function $V: \mathcal{Q} \rightarrow \mathbb{R}_{+}$defined by $V\left(q_{i}\right)=\bar{V}(i)-\min _{i} \bar{V}(i)$ is non-negative and satisfies (19).

Remark 7: A dissipation inequality as in (20) can also be readily verified for $M$; the corresponding network $G_{M}$ is then constructed as follows:

$$
\begin{aligned}
\mathcal{N} & :=\{1, \ldots, n\} \\
\mathcal{E} & :=\left\{(i, j) \in \mathcal{N} \times \mathcal{N} \mid \exists u \in \mathcal{U}_{o} \text { such that } q_{j}=f\left(q_{i}, u\right)\right\} \\
c_{i j} & :=\min _{u \in \mathcal{U}_{o} \mid f\left(q_{i}, u\right)=q_{j}}-\mathbf{I}_{\mathcal{Y}-\mathcal{Y}_{o}}\left(g\left(q_{i}, u\right)\right) .
\end{aligned}
$$

\section{Strongly Polynomial Algorithm for Gain Verification}

We begin by describing an adapted version of the Bellman-Ford algorithm [43], the Adapted Shortest Path Algorithm (ASPA), that can be used to verify the nonexistence of a cycle in a connected subgraph of a given graph $G_{M}$. 


\section{Adapted Shortest Path Algorithm (ASPA)}

Input: Network $G_{M}=(\mathcal{N}, \mathcal{E}, c)$, destination node $k$.

(1) Add a new node numbered $n+1$, and edge $(k, n+1)$

with $c_{k, n+1}=0$.

(2) Initialize the distance labels: $d_{0}(n+1)=0$,

$d_{0}(i)=+\infty$, for $i \neq n+1$.

(3) Initialize a set of nodes: $\operatorname{LIST}_{0}=\mathcal{N}$.

(4) At iteration $t+1$ :

(i) For each node $i \in \operatorname{LIST}_{t}$, update the distance label according to the update law:

$$
d_{t+1}(i)=\min _{j \in \theta i}\left\{d_{t}(j)+c_{i j}\right\}
$$

where $\theta(i)=\{j \in \mathcal{N} \mid(i, j) \in \mathcal{E}\}$.

(ii) For each node $i \notin \operatorname{LIST}_{t}$, set $d_{t+1}(i)=d_{t}(i)$.

(iii) Set $\operatorname{LIST}_{t+1}=\left\{l \in \mathcal{N} \mid(l, i) \in \mathcal{E}\right.$ and $d_{t}(i) \neq$ $\left.d_{t+1}(i)\right\}$.

For completeness, the termination properties and computational complexity of this algorithm are discussed in Appendix III.

The proposed Verification Algorithm (VA), detailed below, implements ASPA successively to verify a given dissipation inequality for a DFM $M$ by verifying the nonexistence of negative cost cycles in the corresponding network $G_{M}$.

\section{Verification Algorithm (VA)}

Input: $G_{M}=(\mathcal{N}, \mathcal{E}, c)$.

(1) $\operatorname{Set} \mathcal{N}_{0}=\mathcal{N}, \mathcal{E}_{0}=\mathcal{E}$.

(2) At iteration $t+1$ :

(i) Pick an arbitrary destination node $k$ in $\mathcal{N}_{t}$.

(ii) Run ASPA on $G_{M}=\left(\mathcal{N}_{t}, \mathcal{E}_{t}, c\right)$. If $d_{n+1} \neq d_{n}$, exit.

(iii) Set $\mathcal{N}_{t+1}=\left\{i \in \mathcal{N}_{t} \mid d(i)=+\infty\right\}$,

$\mathcal{E}_{t+1}=\left\{(i, j) \in \mathcal{E}_{t} \mid i, j \in \mathcal{N}_{t+1}\right\}$

If the Verification Algorithm exits when $d_{n+1} \neq d_{n}$, there exists a negative cost cycle. Otherwise, if it terminates when $\mathcal{N}_{t+1}=\emptyset$, the desired dissipation inequality is verified since the network is free of negative cost cycles. The algorithm terminates in finite time, since there are at most $n+1$ iterations, each of which runs ASPA once, and hence terminates in finite time. Let $n_{o}=n=\operatorname{card}\left(\mathcal{N}_{o}\right)$ and let $n_{i}=\operatorname{card}\left(\mathcal{N}_{i}\right), i \geq 1$. The worst case computational complexity of this algorithm is given by $\mathcal{O}\left(n\left(n-n_{1}\right)+n_{1}\left(n_{1}-n_{2}\right)+\cdots+n_{k-1}\left(n_{k-1}-n_{k}\right)\right) \sim$ $\mathcal{O}\left(n^{2}\right)$.

While the gain of a DFM cannot be exactly computed using this algorithm, an upper bound for it can be computed up to any desired level of accuracy $\epsilon$ by running the Verification Algorithm iteratively: An upper bound ${ }^{3}$ and a lower bound for the gain are established and iteratively refined using a bisection algorithm. The number of required iterations of VA grows inversely with the desired level of accuracy $\epsilon$, with the worst case

\footnotetext{
${ }^{3}$ It is fairly straightforward to show that the $\rho / \mu$ gain of a DFM is either infinite or bounded above by $n \mu_{\max } / \rho_{\min }$ where $n=\operatorname{card}(\mathcal{Q}), \mu_{\max }=$ $\max _{y} \mu(y)$ and $\rho_{\min }=\min _{u \mid \rho(u) \neq 0} \rho(u)$.
}

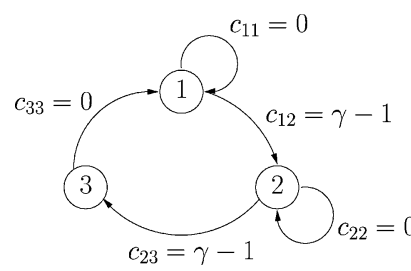

Fig. 6. The network associated with the DFM of Example 7.

complexity of each iteration being $\mathcal{O}\left(n^{2}\right)$. The following simple example illustrates this procedure.

Example 7: Consider once again the deterministic finite state machine $M$ from Example 6, and the corresponding network $G_{M}$ with $\mathcal{N}=\{1,2,3\}, \mathcal{E}=$ $\{(1,1),(1,2),(2,2),(2,3),(3,1)\}$, and costs parametrized by scalar $\gamma$ as follows: $c_{11}(\gamma)=c_{22}(\gamma)=0$, $c_{12}(\gamma)=c_{23}(\gamma)=\gamma-1, c_{33}(\gamma)=\min \{0, \gamma\}$ (Fig. 6). Suppose we wish to verify a gain bound up to desired tolerance $\epsilon=0.1$ : We first set $\gamma=3$ and run VA to verify that $G_{M}$ has no negative cost cycles. Thus, $M$ is $\rho / \mu$ gain stable and 3 is a verified gain bound for it. We then run successive iterations of VA, in which we verify that: $\gamma=1.5$ is a gain bound, $\gamma=0.75$ is not, $\gamma=1.125$ is, $\gamma=0.9375$ is not, $\gamma=1.03125$ is, at which point we stop since a gain bound with the desired level of accuracy has been verified.

\section{CONClusions AND Future Work}

We proposed a framework for robust stability analysis that is particularly applicable for systems over finite alphabets. The framework consists of three notions of input/output stability, and generalized small gain and incremental small gain theorems. We also presented a set of analytical and computational tools for stability analysis of a specific class of models of systems over finite alphabets, namely deterministic finite state machines, proposed as nominal models of more general systems in this framework.

The lack of algebraic structure in finite state machine models gives rise to an interesting problem that is not encountered in the classical robust control framework where the nominal models are LTI. The interconnection of two LTI models of size $n_{1}$ and $n_{2}$ results in an LTI model of size $n_{1}+n_{2}$. The interconnection of two deterministic finite state machine models of size $n_{1}$ and $n_{2}$ results in a deterministic finite state machine of size $n_{1} n_{2}$. This is because the size of an LTI system, for the purpose of analysis (and control), is the dimension of its vector state-space, while that of a deterministic finite state machine model is the cardinality of its state-set. This simple observation motivates the need for approaches that combine component wise analysis with information about the interconnection to guarantee overall performance while avoiding this problem of growth in size of the state-space.

\section{APPENDIX I \\ PROOF OF LEMMA 1}

Proof: The condition given in (21) can be equivalently written as: For every input $\mathbf{u}$ and initial state $q$ there exists a 
finite constant $C_{\mathbf{u}, q}$ such that for all $T \geq 0$, the following inequality holds:

$$
\sum_{t=0}^{T} \sigma(q(t), u(t)) \geq-C_{\mathbf{u}, q}
$$

We claim that $C_{\mathbf{u}, q} \leq n \sigma_{\max }$. The following argument will be needed: If there exists some initial state $q(0)$ and input $\mathbf{u}$ for which $\sum_{t=0}^{T} \sigma(q(t), u(t))<-n \sigma_{\max }$, for some $T$, then $T>n$, because the summation is bounded below by $-T \sigma_{\max }$. Thus there exists two integers $t^{\prime}$ and $t^{\prime \prime}$ in $\mathbb{Z}_{T}, t^{\prime}<t^{\prime \prime}$, such that $q\left(t^{\prime}\right)=q\left(t^{\prime \prime}\right)$. Moreover, it must be the case that $\sum_{t=t^{\prime}}^{t^{\prime \prime}-1} \sigma(q(t), u(t)) \geq 0$, otherwise there exists at least one initial condition and periodic input sequence for which (21) is violated. Let $T^{\prime}=T-\left(t^{\prime \prime}-t^{\prime}\right)$ and consider $\overline{\mathbf{q}} \in \mathcal{Q}^{\mathbb{Z}_{T^{\prime}}}$ and $\overline{\mathbf{u}} \in \mathcal{U}^{\mathbb{Z}_{T^{\prime}}}$ defined by:

$$
\begin{aligned}
& \bar{q}(t):= \begin{cases}q(t), & 0 \leq t<t^{\prime} \\
q\left(t+t^{\prime \prime}-t^{\prime}\right), & t^{\prime} \leq t \leq T^{\prime}\end{cases} \\
& \bar{u}(t):= \begin{cases}u(t), & 0 \leq t<t^{\prime} \\
u\left(t+t^{\prime \prime}-t^{\prime}\right), & t^{\prime} \leq t \leq T^{\prime}\end{cases}
\end{aligned}
$$

Note that $\overline{\mathbf{q}}$ and $\overline{\mathbf{u}}$ are valid state and input sequences, and they satisfy

$$
\sum_{t=0}^{T^{\prime}} \sigma(\bar{q}(t), \bar{u}(t)) \leq \sum_{t=0}^{T} \sigma(q(t), u(t))<-n \sigma_{\max }
$$

Now, suppose our claim that $C_{\mathbf{u}, q} \leq n \sigma_{\max }$ is not true. That is, there exists some initial state $q(0)$ and input $\mathbf{u}$ for which $\sum_{t=0}^{T_{0}} \sigma(q(t), u(t))<-n \sigma_{\max }$, for some $T_{0}$. By the above argument, we can construct a finite sequence of integers $T_{0}>\cdots>T_{k_{o}}$ with corresponding state and input sequences $\mathbf{q}_{k} \in \mathcal{Q}^{\mathbb{Z}_{T_{k}}}$ and $\mathbf{u}_{k} \in \mathcal{U}^{\mathbb{Z}_{T_{k}}}$, satisfying $\sum_{t=0}^{T_{k}} \sigma\left(q_{k}(t), u_{k}(t)\right)<-n \sigma_{\max }$, and such that $T_{k_{o}} \leq n$, and such that $T_{k_{o}} \leq n$, leading to a contradiction.

\section{APPENDIX II}

\section{PROOF OF LEMMA 3}

Proof: By summing up (26) along any trajectory from $t=$ 0 to $t=T$, we get

$$
\begin{aligned}
\sum_{t=0}^{T} \mathbf{I}_{\mathcal{Y}-\mathcal{Y}_{o}}(g(q(t), u(t))) & \leq V(q(0))-V(q(T+1)) \\
& \leq \max _{q_{1}, q_{2}} V\left(q_{1}\right)-V\left(q_{2}\right)
\end{aligned}
$$

Now consider $\mathbf{u} \in \mathcal{U}^{\mathbb{Z}_{\tau-1}}$ and corresponding $\mathbf{q} \in \mathcal{Q}^{\mathbb{Z} \tau}$ satisfying $q(0)=q(\tau)$. First, note that (27) holds for $\tau \leq \operatorname{card}(\mathcal{Q})$ : If $u(t) \in \mathcal{U}-\mathcal{U}_{o}$ for at least one $t$ in $\mathbb{Z}_{\tau-1}$, (27) is clearly satisfied. Otherwise, if $u(t) \in \mathcal{U}_{o}$ for all $t \in \mathbb{Z}_{\tau-1}$, we conclude that $\sum_{t=0}^{\tau-1} \mathbf{I}_{\mathcal{Y}-\mathcal{Y}_{o}}(g(q(t), u(t)))=0$. For if that was not the case, we can construct a periodic input sequence for which the summation $\sum_{t=0}^{T} \mathbf{I}_{\mathcal{Y}-\mathcal{Y}_{o}}(g(q(t), u(t)))$ can be made infinite, thus violating (37) and leading to a contradiction. Next, note that if
$\tau>\operatorname{card}(\mathcal{Q})$, there exists integers $t^{\prime}, t^{\prime \prime}$ in $\mathbb{Z}_{\tau}$ with $t^{\prime}<t^{\prime \prime}$ such that $q\left(t^{\prime}\right)=q\left(t^{\prime \prime}\right), t^{\prime \prime}-t^{\prime} \leq \operatorname{card}(\mathcal{Q})$ and

$$
\sum_{t=t^{\prime}}^{t^{\prime \prime}-1} \gamma \mathbf{I}_{\mathcal{U}-\mathcal{U}_{o}}(u(t))-\mathbf{I}_{\mathcal{Y}-\mathcal{Y}_{o}}(g(q(t), u(t))) \geq 0
$$

by the previous argument. Let $\tau^{\prime}=\tau-\left(t^{\prime \prime}-t^{\prime}\right)$ and define

$$
\begin{aligned}
q^{\prime}(t) & := \begin{cases}q(t), & 0 \leq t<t^{\prime} \\
q\left(t+t^{\prime \prime}-t^{\prime}\right), & t^{\prime} \leq t \leq \tau^{\prime}\end{cases} \\
u^{\prime}(t): & = \begin{cases}u(t), & 0 \leq t<t^{\prime} \\
u\left(t+t^{\prime \prime}-t^{\prime}\right), & t^{\prime} \leq t \leq \tau^{\prime}\end{cases}
\end{aligned}
$$

Note that

$$
\begin{aligned}
& \sum_{t=0}^{\tau-1} \gamma \mathbf{I}_{\mathcal{U}-\mathcal{U}_{o}}(u(t))-\mathbf{I}_{\mathcal{Y}-\mathcal{Y}_{o}}(g(q(t), u(t))) \\
& \quad \geq \sum_{t=0}^{\tau^{\prime}-1} \gamma \mathbf{I}_{\mathcal{U}-\mathcal{U}_{o}}\left(u^{\prime}(t)\right)-\mathbf{I}_{\mathcal{Y}-\mathcal{Y}_{o}}\left(g\left(q^{\prime}(t), u^{\prime}(t)\right)\right) .
\end{aligned}
$$

Thus for any $\tau_{0}>\operatorname{card}(\mathcal{Q})$ we can construct, as described above, a sequence of integers $\tau_{0}, \ldots, \tau_{k_{o}}$ and corresponding state and input sequences $\mathbf{q}_{k} \in \mathcal{Q}^{\mathbb{Z}_{\tau_{k}}}, \mathbf{u}_{k} \in \mathcal{U}^{\mathbb{Z}_{\tau_{k}}}$, with $\tau_{0}>$ $\ldots>\operatorname{card}(\mathcal{Q}) \geq \tau_{k_{o}}$ and such that

$$
\begin{gathered}
\sum_{t=0}^{\tau_{k}-1} \gamma \mathbf{I}_{\mathcal{U}-\mathcal{U}_{o}}\left(u_{k}(t)\right)-\mathbf{I}_{\mathcal{Y}-\mathcal{Y}_{o}}\left(g\left(q_{k}(t), u_{k}(t)\right)\right) \\
\geq \sum_{t=0}^{\tau_{k+1}-1} \gamma \mathbf{I}_{\mathcal{U}-\mathcal{U}_{o}}\left(u_{k+1}(t)\right) \\
\quad-\mathbf{I}_{\mathcal{Y}-\mathcal{Y}_{o}}\left(g\left(q_{k+1}(t), u_{k+1}(t)\right)\right)
\end{gathered}
$$

holds for $k \in\left\{0, \ldots, k_{o}-1\right\}$. It follows that (27) is also satisfied for $\tau>\operatorname{card}(\mathcal{Q})$.

\section{APPENDIX III}

\section{TERMINATION PROPERTIES AND COMPLEXITY OF ASPA}

The termination properties of the Adapted Shortest Path Algorithm are as follows:

(a) If $d_{n+1}=d_{n}$, we have

$$
d_{n+1}(i)=\min _{j \in \theta(i)}\left\{d_{n}(j)+c_{i j}\right\} \leq d_{n+1}(j)+c_{i j}, \forall(i, j) \in \mathcal{E}
$$

Thus, $d_{n+1}$ satisfies (34). Let $\overline{\mathcal{N}}=\left\{i \in \mathcal{N} \mid d_{n+1}(i)=+\infty\right\}$. $\overrightarrow{\mathcal{N}}$ is the set of nodes for which no feasible path exists to node $n+1$ and hence $k$, since if such a path was to exist, it would consist of at most $n$ edges. For every $i \in \mathcal{N}-\overline{\mathcal{N}}$, it follows from Lemma 5 that $d_{n+1}(i)$ is the shortest path length, and hence no negative cost cycles exist in $\mathcal{N}-\overline{\mathcal{N}}$. No conclusions can be drawn about the cost of cycles in $\overline{\mathcal{N}}$.

(b) If $d_{n+1} \neq d_{n}$, a negative cost cycle exists: For if that was not the case, $d_{n}$ would be the shortest path distances, since 
all paths consist of at most $n$ edges, and hence we would have $d_{n}=d_{k}$, for all $k>n$.

Note that the algorithm terminates in at most $n+1$ iterations. Moreover, at each iteration after the first, each edge in $\mathcal{N}-\mathcal{N}_{o}$ is examined at most once. Let $\bar{n}=\operatorname{card}(\overline{\mathcal{N}})$ and assume there are at most $a$ outgoing edges from each node, with $a \ll n$. In this case, the worst case computational complexity is $\mathcal{O}(n a(n-$ $\bar{n})+a n) \sim \mathcal{O}(n(n-\bar{n}))$.

\section{ACKNOWLEDGMENT}

The first author would like to thank V. D. Blondel and P. A. Parrilo for helpful discussions about network flow problems. The authors would like to thank the Associate Editor and the anonymous reviewers for valuable feedback and suggestions for improving the paper.

\section{REFERENCES}

[1] J. P. Hespanha and A. S. Morse, "Stabilization of nonholonomic integrators via logic-based switching," Automatica, vol. 35, pp. 385-393, 1999.

[2] D. Liberzon and J. P. Hespanha, "Stabilization of nonlinear systems with limited information feedback," IEEE Trans. Autom. Control, vol. 50, pp. 910-915, Jun. 2005.

[3] D. Liberzon and A. S. Morse, "Basic problems in stability and design of switched systems," IEEE Control Syst. Mag., vol. 19, pp. 59-70, 1999.

[4] K. Lygeros, K. H. Johansson, S. N. Simic, Z. Jun, and S. Sastry, "Dynamical properties of hybrid automata," IEEE Trans. Autom. Contr., vol. 48, no. 1, pp. 2-17, Jan. 2003.

[5] N. Elia and S. K. Mitter, "Stabilization of linear systems with limited information," IEEE Trans. Autom. Control, vol. 46, no. 9, pp. 1384-1400, 2001.

[6] H. Ye, A. Michel, and L. Hou, "Stability theory for hybrid dynamical systems," IEEE Trans. Autom. Control, vol. 43, pp. 461-474, Apr. 1998.

[7] R. Decarlo, M. Branicky, S. Pettersson, and B. Lennarston, "Perspectives and results on the stability and stabilizability of hybrid systems," Proc. IEEE, vol. 88, no. 7, pp. 1069-1082, Jul. 2000.

[8] T. Henzinger, P.-H. Ho, and H. Wong-Toi, "Algorithmic analysis of nonlinear hybrid systems," IEEE Trans. Autom. Control, vol. 43, no. 4, pp. 540-544, Apr. 1998.

[9] A. Balluchi, L. Benvenuti, M. DiBenedetto, C. Pinello, and A. Sangiovanni-Vincentelli, "Automotive engine control and hybrid systems: Challenges and opportunities," Proc. IEEE, vol. 88, no. 7, pp. 888-912, Jul. 2000.

[10] S. Engell, S. Kowalewski, C. Schulz, and O. Stursberg, "Continuousdiscrete interactions in chemical processing plants," Proc. IEEE, vol. 88, no. 7, pp. 1050-1068, Jul. 2000.

[11] R. Horowitz and P. Varaiya, "Control design of an automated highway system," Proc. IEEE, vol. 88, no. 7, pp. 913-925, Jul. 2000.

[12] D. Pepyne and C. Cassandras, "Optimal control of hybrid systems in manufacturing," Proc. IEEE, vol. 88, pp. 1108-1123, Jul. 2000.

[13] C. Tomlin, G. J. Pappas, and S. Sastry, "Conflict resolution for air traffic management: A study in multi-agent hybrid systems," IEEE Trans. Autom. Control, vol. 43, no. 4, pp. 509-521, Apr. 1998.

[14] R. Alur, C. Belta, V. Kumar, M. Mintz, G. J. Pappas, H. Rubin, and J. Schug, "Biocomputation: Modeling and analyzing biomolecular networks," Computi. Sci. Eng., vol. 4, no. 1, pp. 20-31, 2002.

[15] R. Ghosh and C. Tomlin, "Lateral inhibition through delta-notch signaling: A piecewise affine hybrid model," in Hybrid Systems: Computation and Control, ser. Lecture Notes in Computer Science, M. D. D. Benedetto and A. Sangiovanni-Vincentelli, Eds. Berlin, Germany: Springer-Verlag, 2001, pp. 232-246, no. 2034.

[16] K. Zhou and J. C. Doyle, Essentials of Robust Control. Upper Saddle River, NJ: Prentice Hall, 1998.

[17] G. E. Dullerud and F. Paganini, A Course in Robust Control Theory: A Convex Approach, ser. Texts in Applied Mathematics. New York: Springer-Verlag, 2000, no. 36.

[18] A. Megretski and A. Rantzer, "System analysis via integral quadratic constraints," IEEE Trans. Autom. Control, vol. 47, no. 6, pp. 819-830, 1997.
[19] G. Zames, "On the input-output stability of time-varying nonlinear feedback systems. Part I: Conditions using concepts of loop gain, conicity and positivity," IEEE Trans. Autom. Control, vol. 11, pp. 228-238, 1966.

[20] V. A. Yakubovich, "The S-procedure in nonlinear control theory," Transl.: 1971, english Translation in Vestnik Leningrad Univ. Math. Vestnik Leningradskovo Universiteta, Seriva Matematika, vol. 4, no. 73-93, pp. 62-11, 1977.

[21] A. Megretski and S. Treil, "Power distribution inequalities in optimization and robustness of uncertain systems," J. Math. Syst., Est., Control, vol. 3, no. 3, pp. 301-319, Mar. 1993.

[22] D. Liberzon and D. Nesic, "Stability analysis of hybrid systems via small-gain theorems," in Hybrid Systems: Computation and Control, ser. Lecture Notes in Computer Science, J. Hespanha and A. Tiwari, Eds. Berlin, Germany: Springer-Verlag, 2006, pp. 421-435.

[23] A. Megretski, "Robustness of finite state automata," in Multidisciplinary Research in Control: The Mohammed Dahleh Symp. 2002, L. Giarre and B. Bamieh, Eds., New York, 2003, pp. 147-160.

[24] D. C. Tarraf, "A Finite State Machine Framework for Robust Analysis and Control of Hybrid Systems," Ph.D. dissertation, Dep. Mech. Eng., Mass. Inst. Technol., Cambridge, MA, Jun. 2006.

[25] C. Belta, A. Bicchi, M. Egerstedt, E. Frazzoli, E. Klavins, and G. J. Pappas, "Symbolic planning and control of robot motion," IEEE Robot. Autom. Mag. - Special Issue on Grand Challenges of Robotics, vol. 14, no. 1, pp. 61-71, 2007

[26] C. Belta, V. Isler, and G. Pappas, "Discrete abstractions for robot planning and control in polygonal environments," IEEE Trans. Robotics, vol. 21 , no. 5, pp. 864-874, 2005.

[27] A. Girard and G. J. Pappas, "Approximation metrics for discrete and continuous systems," IEEE Trans. Autom. Control, vol. 52, no. 5, pp. 782-798, May 2007.

[28] A. Girard and G. Pappas, "Approximate bisimulation relations for constrained linear systems," Automatica, vol. 43, no. 8, pp. 1307-1317, Aug. 2007.

[29] A. Julius, "Approximate abstraction of stochastic hybrid automata," in Hybrid Systems: Computation and Control, ser. Lecture Notes in Computer Science, J. Hespanha and A. Tiwari, Eds. Berlin, Germany: Springer-Verlag, 2006, pp. 318-332, 3927.

[30] P. Tabuada, "Symbolic control of linear systems based on symbolic subsystems," IEEE Trans. Autom. Control, vol. 51, no. 6, pp. 1003-1013, Jun. 2006

[31] P. Tabuada, "Symbolic models for control systems," Acta Informatica, vol. 43, pp. 477-500, 2007.

[32] M. Egerstedt, , N. A. Lynch and B. H. Krogh, Eds., "Behavior based robotics using hybrid automata," in Hybrid Systems: Computation and Control, ser. Lecture Notes in Computer Science. Berlin, Germany: Springer-Verlag, 2000, pp. 103-116, 1790.

[33] J. S. Baras and M. R. James, Robust and Risk-Sensitive Output Feedback Control for Finite State Machines and Hidden Markov Models, University of Maryland, College Park, MD 20742, ISR, Tech. Res. Rep., 1994, pp. 1994-1963.

[34] D. C. Tarraf, A. Megretski, and M. A. Dahleh, , A. Bemporad, A Bicchi, and G. Buttazzo, Eds., "Finite state controllers for stabilizing switched systems with binary sensors," in Hybrid Systems: Computation and Control, ser. Lecture Notes in Computer Science. Berlin, Germany: Springer-Verlag, Apr. 2007, pp. 543-556, 2034,.

[35] H. K. Khalil, Nonlinear Systems, 3rd ed. Upper Saddle River, NJ: Prentice Hall, 2002.

[36] J. C. Willems, "Dissipative dynamical systems - Part I: General theory," Arch. Rotational Mech. Analy., vol. 45, pp. 321-351, 1972.

[37] D. Angeli, "A Lyapunov approach to incremental stability properties," IEEE Trans. Autom. Control, vol. 47, no. 3, pp. 410-421, Mar. 2002.

[38] V. Fromion, S. Monaco, and D. Normand-Cyrot, "The weighted incremental norm approach: From linear to nonlinear $\mathcal{H}_{\infty}$ control," Automatica, vol. 37, no. 10, pp. 1585-1592, 2001.

[39] T. Georgiou, "Differential stability and robust control of nonlinear systems," Math. Control, Signals Syst., vol. 6, no. 4, pp. 289-306, 1993.

[40] B. Ingalls and E. D. Sontag, "A small gain theorem with applications to input/output systems, incremental stability, detectability and interconnections," J. Franklin Inst., vol. 339, no. 2, pp. 211-229, 2002

[41] D. C. Tarraf, A. Megretski, and M. A. Dahleh, "Finite automata approximations with error bounds for systems with quantized actuation and measurement: A case study," in Proc. Conf. Dec. Control, Paradise Island, Bahamas, Dec. 2004, pp. 1436-1441.

[42] R. Lopezlena and J. M. A. Scherpen, "Energy functions for dissipativity-based balancing of discrete-time nonlinear systems," Math. Control, Signals Syst., vol. 18, pp. 345-368, 2006. 
[43] D. Bertsimas and J. N. Tsitsiklis, Introduction to Linear Optimization. Belmont, Massachusetts. New York: Athena Scientific, 1997.

[44] R. K. Ahuja, T. L. Magnanti, and J. B. Orlin, Network Flows: Theory, Algorithms and Applications. Englewood Cliffs, NJ: Prentice Hall, 1993.

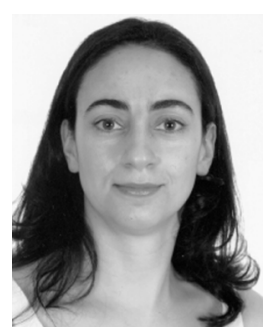

Danielle C. Tarraf (S'01-M'06) received the B.E. degree from the American University of Beirut, Lebanon, in 1996 and the S.M. and Ph.D. degrees from the Massachusetts Institute of Technology (MIT), Cambridge, in 1998 and 2006, respectively, all in mechanical engineering.

She is currently a Postdoctoral Scholar in the Division of Control and Dynamical Systems at the California Institute of Technology, Pasadena. Her research interests include robust analysis and control of hybrid systems, model complexity reduction for control design, and problems at the interface of systems and control theory, automata theory and graph theory.

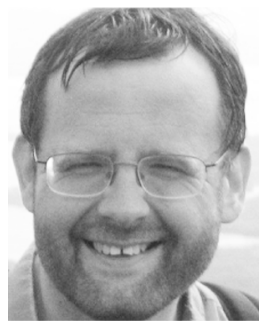

Alexandre Megretski (M'94-SM'08) received the Ph.D. degree in control theory in 1988 from Leningrad University, U.S.S.R.

He was a researcher at the Royal Institute of Technology, Sweden, University of Newcastle, Australia, and a faculty member at Iowa State University, Ames. $\mathrm{He}$ is now a Professor of Electrical Engineering at the Massachusetts Institute of Technology (MIT), Cambridge, working on nonlinear systems (analysis, identification, model reduction, and design) and optimization.

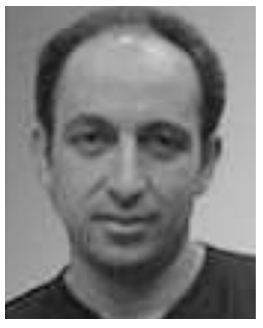

Munther A. Dahleh (S'84-M'87-SM'97-F'01) was born in 1962. He received the B.S. degree from Texas A\&M University, College Station, in 1983, and the Ph.D. degree from Rice University, Houston, TX, in 1987, all in electrical engineering.

Since then, he has been with the Department of Electrical Engineering and Computer Science at MIT, where he is now a full Professor. He is interested in problems at the interface of robust control, filtering, information theory, and computation. $\mathrm{He}$ is also interested in model reduction of discrete-alphabet hidden Markov models, universal learning approaches for systems with both continuous and discrete alphabets, and problems at the interface between systems theory and neurobiology. 